\begin{abstract}
Title of Thesis: $\quad$ OPTIMIZATION OF A PIEZOELECTRIC ACOUSTICAL COMPRESSOR

Degree candidate: Robert A. Dickens

Degree and year: $\quad$ Master of Science, 2004

Thesis directed by: Professor Amr Baz

Department of Mechanical Engineering
\end{abstract}

A one-dimensional, axisymmetric, linear finite element model describing a fluid interacting with a piezoelectric actuator is developed. This system is used to generate finite amplitude standing waves in an acoustic cavity with rigid walls. The model includes the effects of viscous and thermal damping of the fluid at the boundary of the cavity, and material damping in the piezoelectric actuator. Two types of piezoelectric actuators are considered, a stacked layer actuator, and a bending bimorph actuator. The resulting finite element equations are used to determine the optimum shape for the acoustic cavity that results in the highest pressure for the least input power. Optimal chambers were found that could generate \pm 19 psi at $1700 \mathrm{~Hz}$ for 50 watts of power using air as a working fluid and \pm 70 psi at $950 \mathrm{~Hz}$ for 42 watts of power using R-134A as a working fluid. The optimization results were verified against the commercial finite element code ANSYS and published experimental data. 


\title{
OPTIMIZATION OF A PIEZOELECTRIC ACOUSTICAL COMPRESSOR
}

by

\author{
Robert A. Dickens \\ Thesis submitted to the Faculty of the Graduate School of the \\ University of Maryland, College Park in partial fulfillment \\ of the requirements for the degree of \\ Master of Science \\ 2004
}

Advisory Committee:

Professor Amr Baz, Chair/Advisor

Professor Balakumar Balachandran

Associate Professor Donald DeVoe 


\section{TABLE OF CONTENTS}

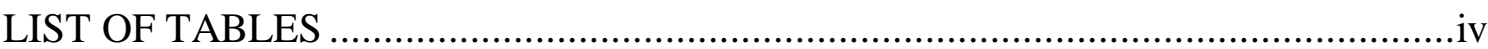

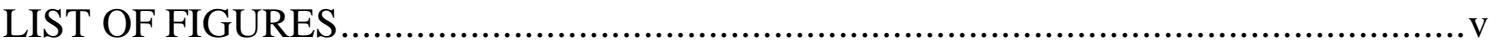

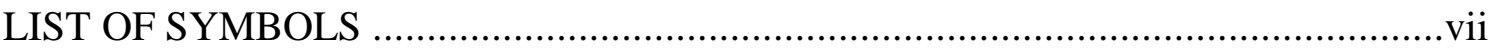

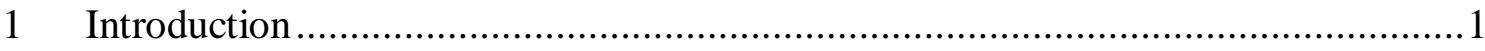

2 Theory

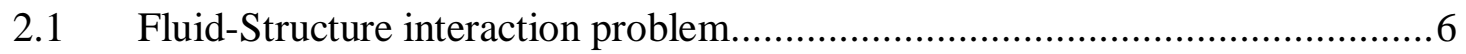

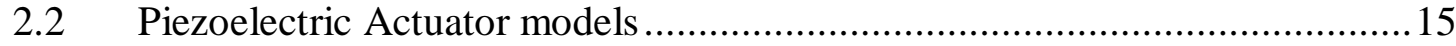

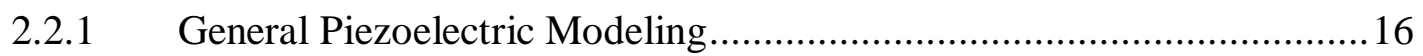

2.2.2 Derivation of FE model of stacked actuator ......................................22

2.2.3 Derivation of FE model for axisymmetric bimorph disc .......................25

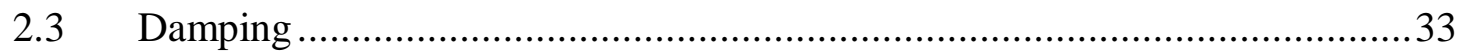

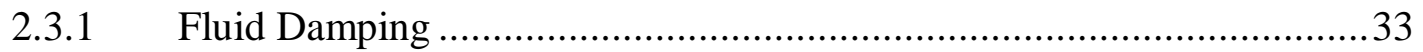

2.3.2 Damping in the piezoelectric actuator................................................... 37

2.4 Implementation and Optimization in Matlab …............................................38

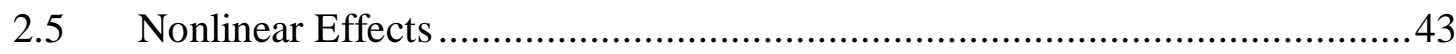

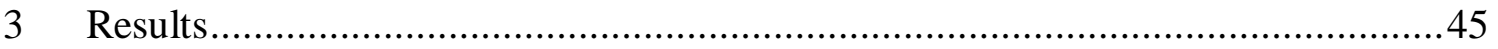

3.1 Verification of Acoustic Fluid FEM and Damping .....................................46

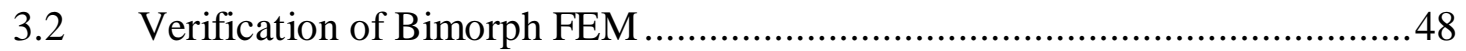

3.3 Verification of Bimorph FEM with fluid and damping in a sample cavity .....50

3.4 Comparison with Published Measurement Data ............................................51

3.5 Optimization of cavity/actuator system ………........................................58 


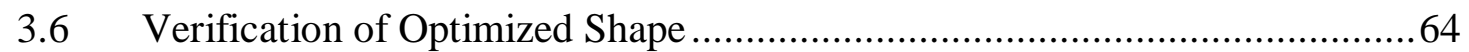

$4 \quad$ Summary, Conclusions, and Future Work ......................................................... 71

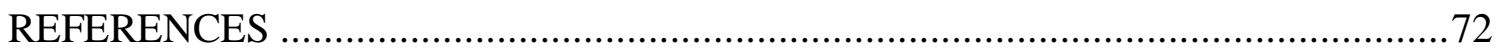




\section{LIST OF TABLES}

Table 1 - Comparison of Types of Acoustical Standing Wave Compressors.................4

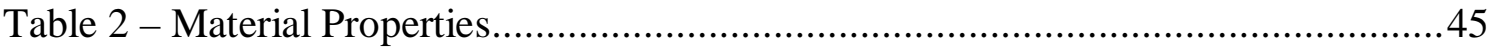

Table 3 - Frequency and End Pressure Comparison of Verification Model Cavity........47

Table 4 - Bimorph Model Frequency and Displacement Comparison........................49

Table 5 - Bimorph Model Drive Power Comparison .............................................. 49

Table 6 -Tapered PAC Model Frequency and End Pressure Comparison .....................50

Table 7 -Tapered PAC Model Displacement and Drive Power Comparison ................51

Table 8 - Material Properties for R-134A used in Experiment ................................52

Table 9 - Comparison of Matlab Linear Code to Experiment ..................................56

Table 10 - Comparison of Matlab Linear Code to Experiment (First Harmonic)...........56

Table 11 - Comparison of Matlab Linear Code to ANSYS for 14.74g Acceleration of

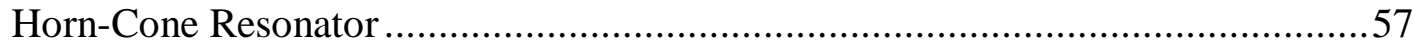

Table 12 - Comparison of Matlab Linear Code to ANSYS for 28.02g Acceleration of

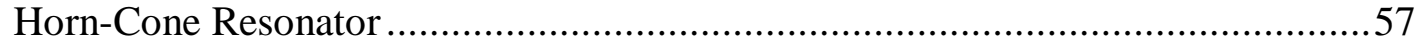

Table 13 - Comparison of Optimized PAC Frequency and Pressure Results (Air) ........67

Table 14 - Comparison of Optimized PAC Displacement and Power Results (Air) ......67

Table 15 - Comparison of Optimized PAC Frequency and Pressure Results (R-134A). 70

Table 16 - Comparison of Optimized PAC Displacement and Power Results (R-134A) 


\section{LIST OF FIGURES}

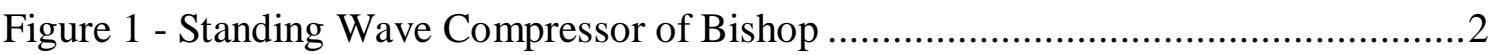

Figure 2 - Standing Wave Compressor of Lucas ......................................................

Figure 3 - Piezoelectric Acoustical Compressor (PAC) Concept ……………….......... 4

Figure 4 - Simplified Diagram of Typical PAC ..................................................6

Figure 5 - Finite Element Model of Acoustic Fluid Cavity .......................................... 9

Figure 6 - Typical Acoustic Fluid Element..............................................................

Figure 7 - Standard Directions in Piezoelectric Material ............................................ 15

Figure 8 - Typical Piezoelectric Actuator Types ......................................................... 16

Figure 9 - Three Noded Stacked Actuator Finite Element ..........................................23

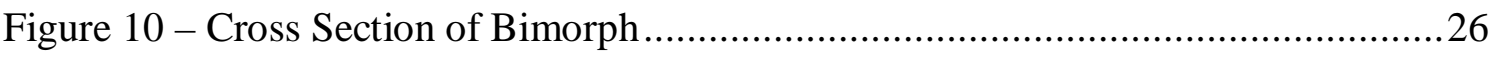

Figure 11 - Typical Axisymmetric Bimorph Finite Element .........................................29

Figure 12 - Finite Element Model of Acoustic Cavity with Bimorph Actuator ..............38

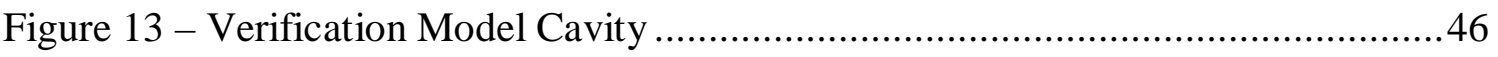

Figure 14 - Comparison of Matlab and ANSYS Solutions of Verification Model Cavity

Figure 15 - Bimorph Results Comparison, Matlab (M) vs. ANSYS (A) .......................49

Figure 16 -MATLAB output for horn-cone geometry using ERD at 14.74g (41.7 Watts)

Figure 17 -MATLAB output for horn-cone geometry using ERD at 28.02g (144 Watts)

Figure 18 - Time History Comparison of MATLAB to Experiment (41.7 Watts) .........55 
Figure 19 - Time History Comparison of MATLAB to Experiment (144 Watts) ..........55

Figure 20 - Matlab output of optimized shape (Air) ..........................................59

Figure 21 - Matlab frequency response of optimized shape (Air) ...........................6 60

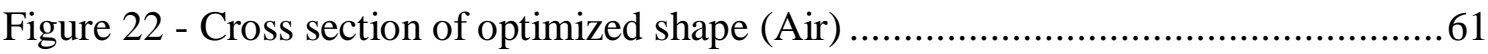

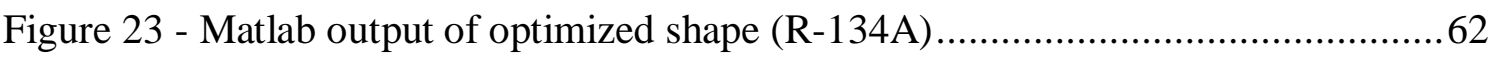

Figure 24 - Matlab frequency response of optimized shape (R-134A) ......................63

Figure 25 - Mode 1 and 2 Pressure (psi) in optimized cavity (ANSYS, Air)...............65

Figure 26 - Modes 3 and 4 Pressure (psi) in optimized cavity (ANSYS, Air) ..............66

Figure 27 - Mode 1 and 2 Pressure (psi) in optimized cavity (ANSYS, R-134A)........68

Figure 28 - Mode 3 and 4 Pressure (psi) in optimized cavity (ANSYS, R-134A)........69 


\section{LIST OF SYMBOLS}

\begin{tabular}{|c|c|}
\hline$A$ & Cross sectional area \\
\hline$a$ & Imposed acceleration on the resonator \\
\hline$a_{1}, a_{2}, a_{3}$ & Interpolation constants \\
\hline$B / A$ & Nonlinearity parameter of a fluid \\
\hline$B_{u}$ & Matrix that relates strain to displacement, geometry dependent \\
\hline$C$ & Capacitance \\
\hline$C_{p}$ & Specific heat at constant pressure \\
\hline$C R$ & Piezoelectric reduced damping matrix \\
\hline$C_{s}$ & Structure damping matrix \\
\hline$C_{u u}$ & Piezoelectric viscous damping matrix \\
\hline$c_{i j}^{E}$ & Piezoelectric compliance coefficient in direction $i j$ at constant electric field \\
\hline$c$ & Thermodynamic speed of sound in the fluid \\
\hline$c^{*}$ & Complex speed of sound \\
\hline$D_{i}$ & Electric displacement in direction $i$ \\
\hline$d_{33}$ & Piezoelectric strain constant \\
\hline$E_{i}$ & Electric field in direction $i$ \\
\hline$e_{i j}$ & Piezoelectric constant in direction $i j$ \\
\hline$F$ & Structural force vector \\
\hline$F R$ & Piezoelectric reduced force matrix \\
\hline$f$ & Frequency \\
\hline$f_{s i}$ & Fluid-structure interaction node \\
\hline
\end{tabular}




\begin{tabular}{|c|c|}
\hline$\{h\}$ & Interpolating vector \\
\hline$I$ & Current \\
\hline$K^{*}$ & Complex stiffness \\
\hline$K_{f}$ & Fluid stiffness matrix \\
\hline$K_{s}$ & Structure stiffness matrix \\
\hline$K_{u u}$ & Piezoelectric Structure stiffness matrix \\
\hline$K_{u d}$ & Piezoelectric electromechanical coupling matrix \\
\hline$K_{d d}$ & Piezoelectric electric displacement stiffness matrix \\
\hline$K R$ & Piezoelectric reduced stiffness matrix \\
\hline$M_{f}$ & Fluid mass matrix \\
\hline$M_{s}$ & Structural mass matrix \\
\hline$M_{u u}$ & Piezoelectric mass matrix \\
\hline$M R$ & Piezoelectric reduced mass matrix \\
\hline$N_{u}$ & Structural displacement interpolation vector \\
\hline$N_{1}, N 2$ & Non-linear fluid matrices \\
\hline$n$ & Number of layers in stacked piezoelectric actuator \\
\hline$P$ & Electrical power \\
\hline$p$ & Fluid pressure \\
\hline$Q$ & Surface charge \\
\hline$Q_{M}$ & Mechanical amplification factor \\
\hline$Q_{n c}$ & Non-conservative force \\
\hline$q$ & Vector of generalized coordinates \\
\hline$r_{i}$ & Radius of node $i$ \\
\hline
\end{tabular}




$\begin{array}{ll}S_{i} & \text { Piezoelectric mechanical strain in direction } i \\ T & \text { Surface area } \\ T_{i} & \text { Kinetic energy } \\ t & \text { Piezoelectric mechanical stress in direction } I \\ \tan \delta & \text { Loss tangent } \\ U & \text { Potential energy } \\ \{u\} & \text { Structure displacement vector } \\ V_{0} & \text { Voltage } \\ v & \text { Fluid particle velocity } \\ W_{n c} & \text { Virtual work due to non-conservative forces } \\ w(x) & \text { Mid-plane deflection in the axial direction } \\ z & \text { Coordinate along the thickness of the element }\end{array}$

\section{Greek Symbols}

$\alpha \quad$ Absorption coefficient

$\beta \quad$ Acoustic boundary absorption coefficient

$\gamma \quad$ Ratio of specific heats

$\Delta L \quad$ Change in length

$\delta \quad$ Variational indicator

$\Delta \quad$ General nodal deflection vector

$\Delta_{u} \quad$ Piezoelectric element nodal mechanical displacement vector

$\Delta_{d} \quad$ Piezoelectric element nodal electrical displacement vector 
$\varepsilon_{i j} \quad$ Permittivity matrix in direction $i j$ at constant stress

$\phi \quad$ Fluid velocity potential

$\{\Phi\} \quad$ Fluid element nodal deflection vector

$\kappa \quad$ Thermal conductivity of the fluid

$\eta \quad$ Coefficient of shear viscosity

$\eta_{e} \quad$ Effective coefficient of shear viscosity

$\eta_{L} \quad$ Loss factor

$\Omega \quad$ Fluid-structure interaction matrix

$\rho \quad$ Mass density

$\omega \quad$ Circular frequency

$\zeta \quad$ Viscous damping ratio

Abbreviations

ERD Entire Resonator Drive

FEM Finite Element Method

PAC Piezoelectric Acoustical Compressor 


\section{Introduction}

This thesis was inspired by the work of Lucas (1994) and Bishop (2000) who hold patents for acoustical compressors based on generating standing waves in a cavity. Lucas used a specially shaped resonator chamber driven by an electromechanical actuator, while Bishop used a piezoelectric actuator driving the fluid within a straight tube. Baz (2000) combined these two ideas to create a new type of acoustical compressor. It consists of a specially shaped chamber with the fluid driven directly by a piezoelectric actuator. The purpose of this thesis is to derive an analytical model describing the pressure in the chamber based on its shape and the size of the actuator. This model is then used to create an optimum shape for the cavity and actuator to maximize the pressure and minimize the power input.

In the case of Bishop's compressor sketched in Figure 1, a piezoelectric actuator is used to generate standing pressure waves in a straight chamber. This has the advantage of using no moving parts for the actuator. The standing wave generated by the actuator creates negative and positive pressure during each acoustic cycle. The valves allow the working fluid to be drawn into the chamber during the negative pressure portion of the cycle and delivered to the system during the positive pressure portion. The pressure developed in a straight tube is considerably less than the pressure that can be created in a shaped chamber. 


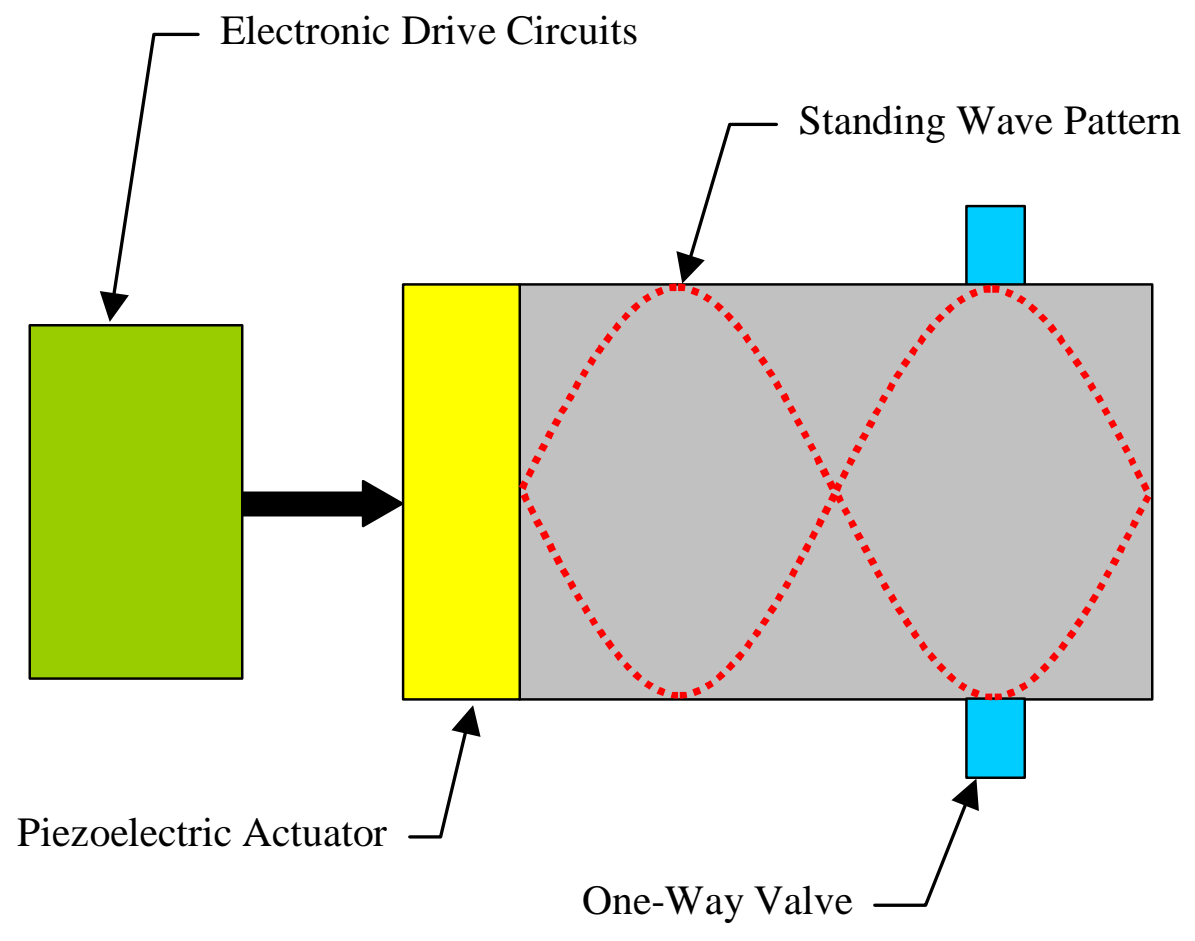

Figure 1 - Standing Wave Compressor of Bishop

A simple representation of Lucas' compressor is sketched in Figure 2. A conventional reciprocating electro-mechanical actuator is used to oscillate a specially shaped chamber. This drive method makes virtually all of the interior surface area of the resonator available for driving the working fluid. Lucas collaborated with other researchers in publishing two papers (Lawrenson, et al., 1998; Ilinskii, et al., 1998), which describe the experimental results and theory. Ilinskii et al. (1998) created a one-dimensional, time domain model using the gas dynamics equations to calculate non-linear standing waves in an oscillating chamber with an imposed acceleration. The solution of the nonlinear equations is beyond the scope of this thesis and seeking this solution would have increased computation time significantly during optimization. However, the 
experimental results of Lawrenson, et al. (1998) may be used as a point of reference to evaluate the utility of the results obtained from the linear equations.

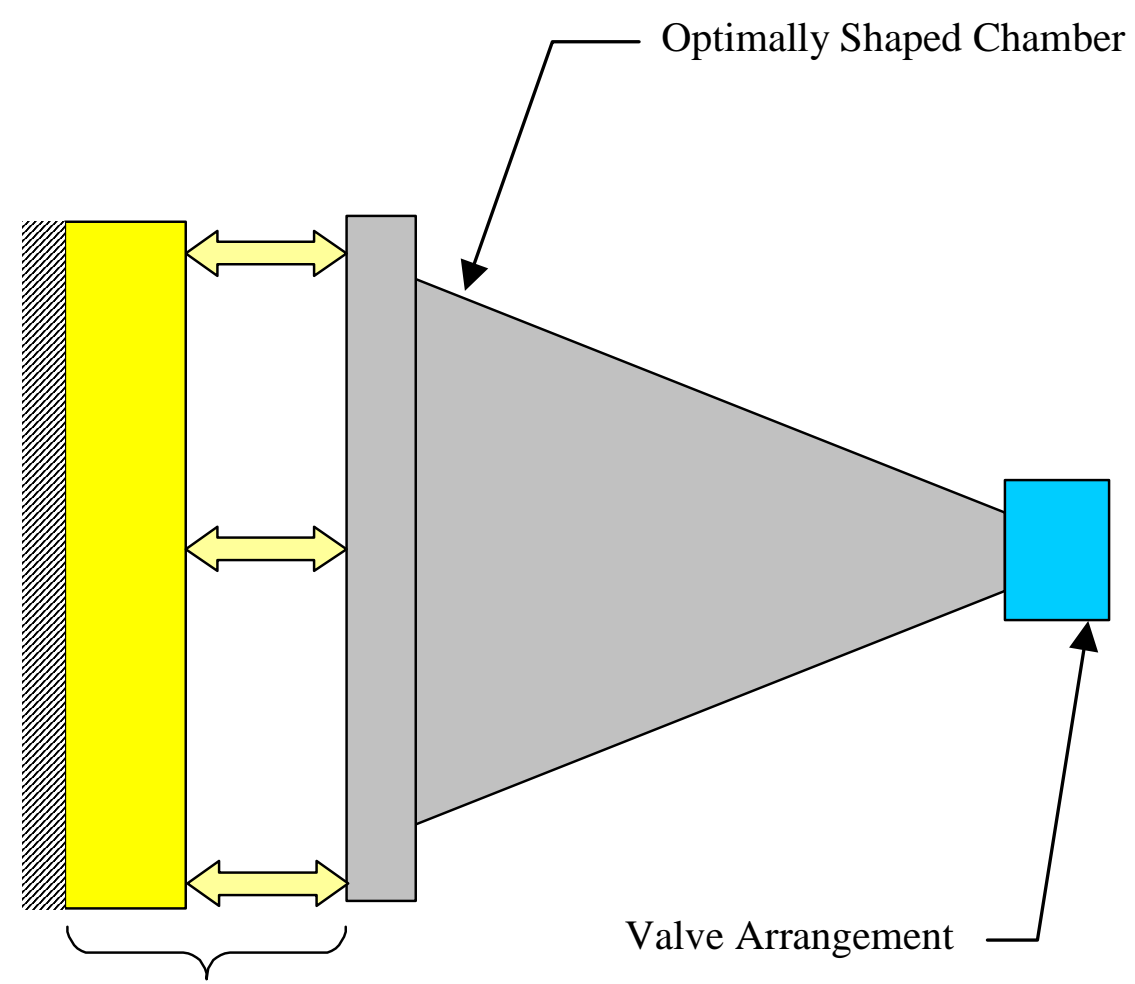

Reciprocating Actuator

Figure 2 - Standing Wave Compressor of Lucas

In Lucas' approach, high pressures can be generated but a conventional reciprocating actuator with moving parts is used. Bishop's compressor uses a piezoelectric actuator but it does not generate useful pressure. The design concept shown in Figure 3 combines the simplicity of a stationary chamber and a piezoelectric actuator with the high pressures attainable with a shaped chamber. A summary of the three designs of standing wave compressors is given in Table 1. 


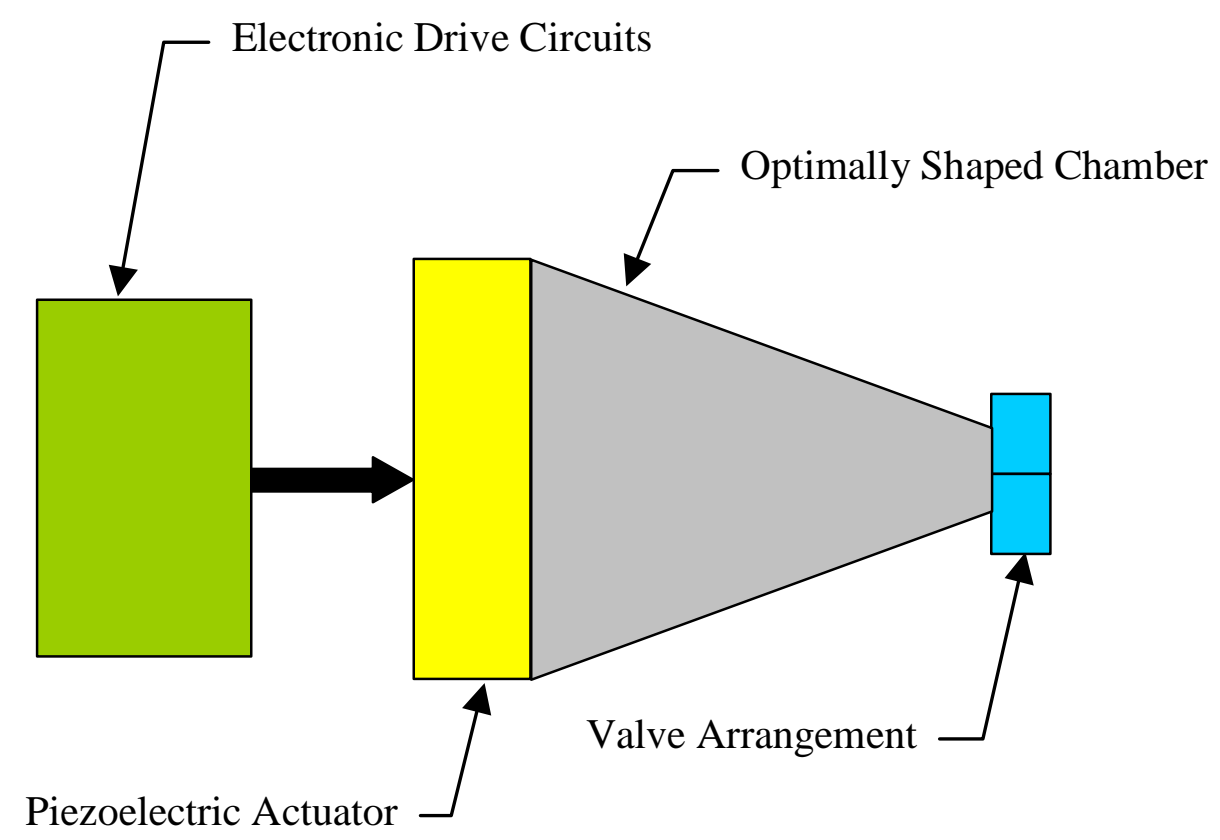

Figure 3 - Piezoelectric Acoustical Compressor (PAC) Concept

Table 1 - Comparison of Types of Acoustical Standing Wave Compressors

\begin{tabular}{|c|l|l|}
\hline Compressor Type & \multicolumn{1}{|c|}{ Advantages } & \multicolumn{1}{c|}{ Disadvantages } \\
\hline Bishop & $\begin{array}{l}\text { Piezoelectric actuator } \\
\text { Stationary chamber }\end{array}$ & $\begin{array}{l}\text { Low pressures } \\
\text { Small surface area for power } \\
\text { transfer to the working fluid }\end{array}$ \\
\hline Lucas & $\begin{array}{l}\text { High Pressures } \\
\text { Large surface area for power } \\
\text { transfer to the working fluid }\end{array}$ & Conventional actuator \\
Moving chamber
\end{tabular}


The equations of motion of the system must include some interesting features such as fluid-structure interaction, coupled electrical and mechanical fields, and acoustic fluid elements. It is the purpose of this thesis to create a linear mathematical model of a finite amplitude standing wave compressor with a piezoelectric actuator. This model will then be used to create an optimally shaped chamber, which produces the maximum pressure at the end opposite to the actuator and requires the least amount of electrical input power. 


\section{Theory}

The Finite Element Method (FEM) is used to model the acoustical compressor. The problem consists of a fluid domain, an actuator, and the interaction between the two. First, the development of the FEM matrices for the fluid and a generic actuator is considered. The actuator model is then expanded from a simple mass/damper/spring system as shown in Figure 4 into a piezoelectric actuator. The inclusion of the actuator dynamics in the model allows for simultaneous optimization of the cavity and the drive system.

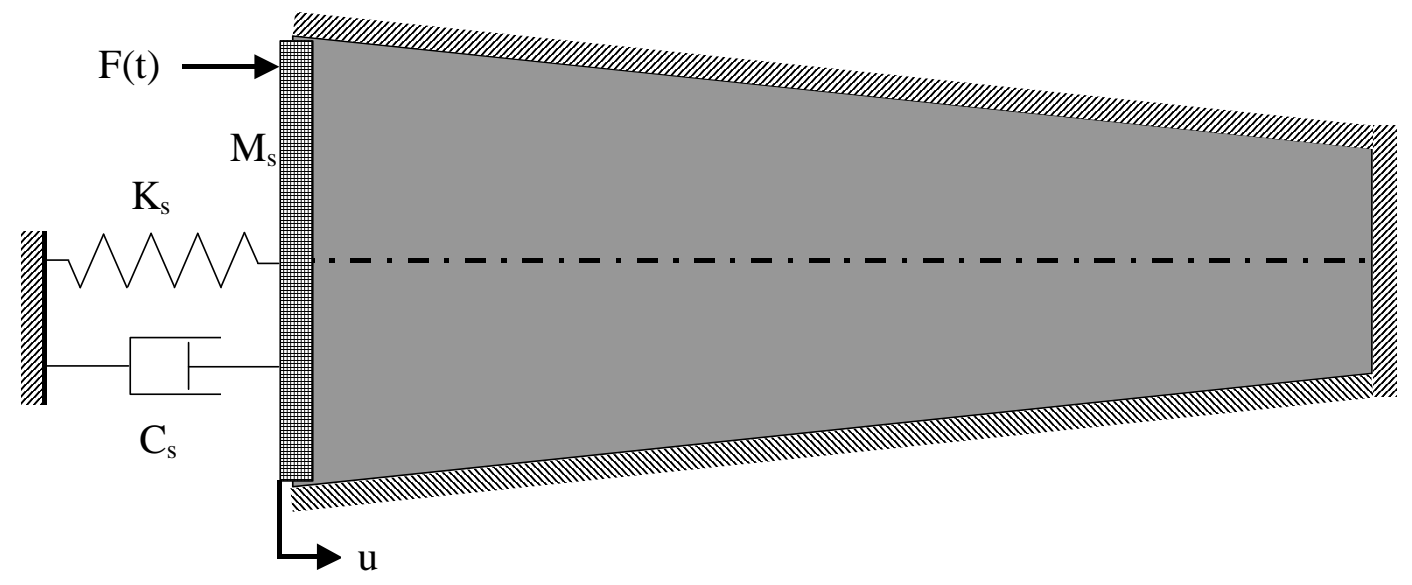

Figure 4 - Simplified Diagram of Typical PAC

\subsection{Fluid-Structure interaction problem}

The acoustic fluid in the cavity is modeled using the approach developed by Everstine (1981), Olson and Bathe (1985), and Bathe (1996). This procedure assumes an inviscid, 
isotropic fluid undergoing small displacements and having no body forces. The momentum equation under these conditions simplifies to

$$
\rho \dot{v}+\nabla p=0
$$

where $\rho=$ mass density, $v=$ particle velocity, and $p=$ pressure. A velocity potential function $\phi$ is defined such that

$$
v=\nabla \phi
$$

Solving the momentum equation for pressure gives

$$
p=-\rho \dot{\phi}
$$

The equations of motion will be generated using the Hamilton Principle (Meirovich, 2001). This approach requires the calculation of Kinetic $(T)$ and Potential $(U)$ energies, and virtual work due to non-conservative forces $\left(W_{n c}\right)$.

$$
\int_{t_{1}}^{t_{2}}\left(\delta T-\delta U+\overline{\delta W_{n c}}\right) d t=0
$$

where $\delta$ indicates variation in the generalized coordinates.

The energies of the fluid $(f)$ and structure $(s)$ are (Baz, 1997)

$$
\begin{gathered}
T_{f}=1 / 2 \int_{V} m v^{2} d V=1 / 2 \int_{0}^{L} \rho A(x)(\nabla \phi)^{2} d x, \\
T_{s}=1 / 2 \int_{V} M_{s} \dot{u}^{2} d V, \\
U_{f}=1 / 2 \int_{V} \frac{P^{2}}{\rho c^{2}} d V=1 / 2 \int_{0}^{L} \frac{1}{c^{2}} \rho A(x) \dot{\phi}^{2} d x, \\
U_{s}=1 / 2 \int_{V} K_{s} u^{2} d V
\end{gathered}
$$

and 
where subscript $f$ refers to the fluid, subscript $s$ refers to the structure, $\mathrm{V}=$ volume, $c=$ speed of sound in the fluid, and $p=-\rho \dot{\phi}$ has been substituted for the pressure.

The final equations needed are the work done due to the non-conservative forces acting on the system. These forces are the actuation force on the structure $(s)$, the force due to the damper $(d)$, and the fluid-structure interaction forces $(f s i)$

and

$$
\begin{gathered}
F_{s}=F(t), \\
F_{d}=-C_{s} \dot{u}, \\
F_{f s i}=-P A=\rho \dot{\phi}_{f s i} A_{f s i}
\end{gathered}
$$

where $f s i$ indicates the locations in the fluid that interact with the structure. The area $(A)$ is the projection of the interaction area in the direction of interest. For the onedimensional model considered here, this direction is along the resonator axis. From these equations, the variation in the virtual work is

$$
\overline{\delta W_{n c}}=F_{s} \delta u-C_{s} \dot{u} \delta u+\rho \dot{\phi}_{f s i} A_{f s i} \delta u_{f s i}+\rho A_{f s i} u_{f s i} \delta \dot{\phi}_{f s i}
$$

where the variation is taken with respect to the general coordinates. Hamilton's principle for the system under consideration can now be written as

$$
\begin{gathered}
\int_{t_{1}}^{t_{2}} \delta\left(T_{s}+T_{f}-U_{s}-U_{f}\right) d t \\
+\int_{t_{1}}^{t_{2}}\left(F_{s} \delta u-C_{s} \dot{u} \delta u+\rho A_{f s i} \dot{\phi}_{f s i} \delta u_{f s i}+\rho A_{f s i} u_{f s i} \delta \dot{\phi}_{f s i}\right) d t=0
\end{gathered}
$$

Equation 9 along with equations 5 and 6 describe the governing equations for the system. We are now in a position to form the finite element equations for the system. 
The discretized fluid cavity is shown below in Figure 5. A typical three noded, onedimensional, fluid finite element in the cavity is shown in Figure 6.

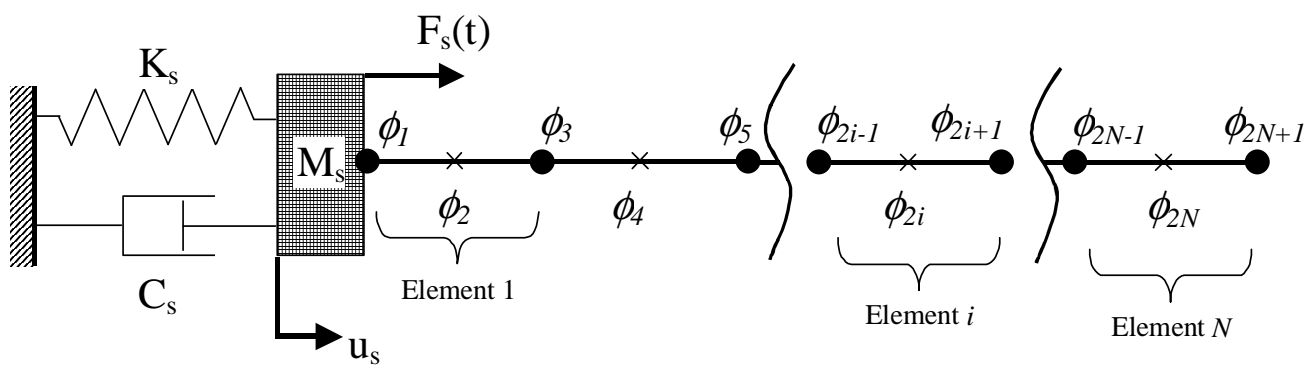

Figure 5 - Finite Element Model of Acoustic Fluid Cavity

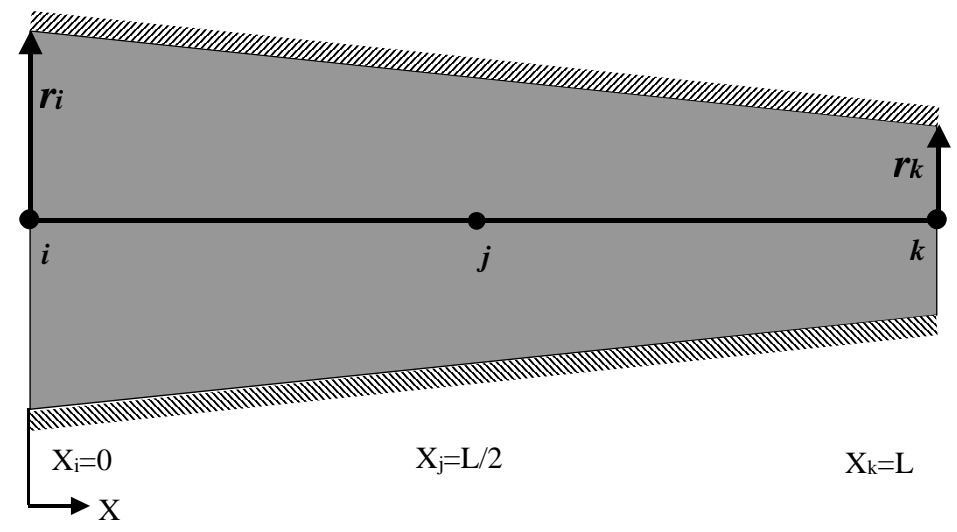

Figure 6 - Typical Acoustic Fluid Element

The value of the potential function at a point along the element is assumed to vary quadratically (Bathe 1996). The fluid potential can thus be written as

$$
\begin{gathered}
\phi(x)=a_{1}+a_{2} \cdot x+a_{3} \cdot x^{2} \text {; or in matrix form } \\
\phi(x)=\left(\begin{array}{lll}
1 & x & x^{2}
\end{array}\right)\left(\begin{array}{lll}
a_{1} & a_{2} & a_{3}
\end{array}\right)^{T}
\end{gathered}
$$

subject to the boundary conditions

$$
\phi(0)=\phi_{i} ; \phi\left(\frac{L}{2}\right)=\phi_{j} ; \phi(L)=\phi_{k}
$$


Combining equations 10 and 11 into a matrix equation yields the fluid element nodal deflection vector

$$
\{\Phi\}=\left\{\begin{array}{l}
\phi_{i} \\
\phi_{j} \\
\phi_{k}
\end{array}\right\}=\left[\begin{array}{ccc}
1 & 0 & 0 \\
1 & \frac{L}{2} & \left(\frac{L}{2}\right)^{2} \\
1 & L & L^{2}
\end{array}\right]\left\{\begin{array}{l}
a_{1} \\
a_{2} \\
a_{3}
\end{array}\right\}
$$

Solving equation 12 for the unknown interpolation constants $a_{1}, a_{2}, a_{3}$ and substituting into equation 10 yields:

$$
\begin{gathered}
\left.\phi(x)=\left[\begin{array}{lll}
1 & x & x^{2}
\end{array}\right] \begin{array}{ccc}
1 & 0 & 0 \\
1 & \frac{L}{2} & \left(\frac{L}{2}\right)^{2} \\
1 & L & L^{2}
\end{array}\right]^{-1}\{\Phi\}, \\
\text { i.e. } \phi(x)=\left\{1-3 \frac{x}{L}+2\left(\frac{x}{L}\right)^{2} \quad 4 \frac{x}{L}-4\left(\frac{x}{L}\right)^{2}-\frac{x}{L}+2\left(\frac{x}{L}\right)^{2}\right\}\{\Phi\}
\end{gathered}
$$

Equation 14 can be written symbolically as $\phi(x)=\{h\} \Phi \Phi\}$ where $\{h\}$ is the $1 \mathrm{x} 3$ interpolating vector. With this definition of the velocity potential, the particle velocity is now given by

$$
\left.v=\phi_{x}=\left\{h_{x}\right\} \Phi\right\}
$$

The cross sectional area for the tapered fluid element shown in Figure 6 is given by

where

$$
\begin{gathered}
A(x)=\pi r(x)^{2} \\
r(x)=r_{i}+\left(r_{j}-r_{i}\right) \frac{x}{L}
\end{gathered}
$$

Recalling equation 5, the fluid and structure kinetic energies in terms of the nodal potentials and the structure displacement vector $u$ are 


$$
\begin{aligned}
T_{f} & \left.=1 / 2 \int_{0}^{L} \rho A(x)\{\Phi\}^{T}\left\{h_{x}\right\}^{T}\left\{h_{x}\right\} \Phi\right\} d x \\
& =1 / 2\{\Phi\}^{T}\left[\int_{0}^{L} \rho A(x)\left\{h_{x}\right\}^{T}\left\{h_{x}\right\} d x\right]\{\Phi\},
\end{aligned}
$$

and

$$
T_{s}=1 / 2 \int_{V} M_{s} \dot{u}^{2} d V=1 / 2\{\dot{u}\}^{T}\left[M_{s}\right]\{\dot{u}\}
$$

The fluid kinetic energy may be further simplified as

where

$$
\begin{gathered}
T_{f}=1 / 2\{\Phi\}^{T}\left[K_{f}\right]\{\Phi\}, \\
{\left[K_{f}\right]=\int_{0}^{L} \rho A(x)\left\{h_{x}\right\}^{T}\left\{h_{x}\right\} d x}
\end{gathered}
$$

Similarly, the potential energies may be written as

$$
\begin{aligned}
& U_{f}=1 / 2 \frac{1}{c^{2}} \int_{0}^{L} \rho A(x)\{\dot{\Phi}\}^{T}\{h\}^{T}\{h\}\{\dot{\Phi}\} d x \\
& =1 / 2\{\dot{\Phi}\}^{T}\left[\int_{0}^{L} \frac{1}{c^{2}} \rho A(x)\{h\}^{T}\{h\} d x\right]\{\dot{\Phi}\}, \\
& \text { and } \quad U_{s}=1 / 2 \int_{V} K_{s} u^{2} d V=1 / 2\{u\}^{T}\left[K_{s}\right]\{u\}
\end{aligned}
$$

Simplifying the fluid potential energy yields

where

$$
\begin{gathered}
U_{f}=1 / 2\{\dot{\Phi}\}^{T}\left[M_{f}\right][\dot{\Phi}\}, \\
{\left[M_{f}\right]=\int_{0}^{L} \frac{1}{c^{2}} \rho A(x)\{h\}^{T}\{h\} d x}
\end{gathered}
$$

Substituting the kinetic energy, potential energy, and the virtual work in equation 9 and expanding the coordinates to nodal coordinates yields 


$$
\begin{gathered}
\int_{t_{1}}^{t_{2}} \delta\left(1 / 2\{\dot{u}\}^{T}\left[M_{s}\right]\{\dot{u}\}+1 / 2\{\Phi\}^{T}\left[K_{f}\right]\{\Phi\}\right) d t \\
-\int_{t_{1}}^{t_{2}} \delta\left(1 / 2\{u\}^{T}\left[K_{s}\right]\{u\}+1 / 2\{\dot{\Phi}\}^{T}\left[M_{f}\right]\{\dot{\Phi}\}\right) d t \\
+\int_{t_{1}}^{t_{2}}\left(F_{s} \delta\{u\}-C_{s} \dot{u} \delta\{u\}+\rho A\left\{\dot{\Phi}_{f s i}\right\} \delta\left\{u_{f s i}\right\}+\rho A\left\{u_{f s i}\right\} \delta\left\{\dot{\Phi}_{f s i}\right\}\right) d t=0
\end{gathered}
$$

Taking the variations indicated yields

$$
\begin{gathered}
\int_{t_{1}}^{t_{2}}\left(\left[M_{s}\right]\{\dot{u}\} \delta\{\dot{u}\}+\left[K_{f}\right]\{\Phi\} \delta\{\Phi\}\right) d t \\
\left.\left.-\int_{t_{1}}^{t_{2}}\left(\left[K_{s}\right]\{u\} \delta\{u\}+\left[M_{f}\right]\right\} \dot{\Phi}\right\} \delta\{\dot{\Phi}\}\right) d t \\
+\int_{t_{1}}^{t_{2}}\left(F_{s} \delta\{u\}-C_{s} \dot{u} \delta\{u\}+\rho A\left\{\dot{\Phi}_{f s i}\right\} \delta\left\{u_{f s i}\right\}+\rho A\left\{u_{f s i}\right\} \delta\left\{\dot{\Phi}_{f s i}\right\}\right) d t=0
\end{gathered}
$$

Rearranging terms gives

$$
\begin{gathered}
\underbrace{t_{2}}_{t_{1}}\left(\left[M_{s}\right]\{\dot{u}\} \delta\{\dot{u}\}-\left[M_{f}\right]\{\dot{\Phi}\} \delta\{\dot{\Phi}\}\right) d t \\
\left.+\int_{t_{1}}^{t_{2}}\left(\left[K_{f}\right] \int \Phi\right\} \delta\{\Phi\}-\left[K_{s}\right]\{u\} \delta\{u\}\right) d t \\
\left.+\int_{t_{1}}^{t_{2}}(F_{s} \delta\{u\}-C_{s} \dot{u} \delta\{u\}+\rho A_{f s i}\left\{\dot{\Phi}_{f s i}\right\} \delta\left\{u_{f s i}\right\}+\underbrace{\rho A_{f s i}}_{I I} \underbrace{\left\{u_{f s i}\right\} \delta\{\dot{\Phi} f s i}\}\right) d t=0
\end{gathered}
$$

Integrating the portion of this equation labeled $I$ by parts yields

$$
\begin{aligned}
& I=\left.\left[M_{s}\right]\{\dot{u}\} \delta\{u\}\right|_{t_{1}} ^{t_{2}}-\left.\left[M_{f}\right]\{\dot{\Phi}\} \delta\{\Phi\}\right|_{t_{1}} ^{t_{2}} \\
& -\int_{t_{1}}^{t_{2}}\left(\left[M_{s}\right]\{\ddot{u}\} \delta\{u\}+\left[M_{f}\right]\{\ddot{\Phi}\} \delta\{\Phi\}\right) d t
\end{aligned}
$$


As the virtual displacements are arbitrary, we choose them such that they are zero at $t_{1}$ and $t_{2}$. Thus, $I$ is

$$
I=-\int_{t_{1}}^{t_{2}}\left(\left[M_{s}\right]\{\ddot{u}\} \delta\{u\}+\left[M_{f}\right]\{\ddot{\Phi}\} \delta\{\Phi\}\right) d t
$$

Integrating $I I$ by parts and invoking the arbitrariness of the virtual displacements yields

$$
\begin{aligned}
I I & =\rho A_{f_{s i}}\left\{u_{f s i}\right\} \delta\left\{\Phi_{f s i}\right\}_{t_{1}}^{t_{2}}-\int_{t_{1}}^{t_{2}}\left(\rho A_{f s i}\left\{\dot{u}_{f s i}\right\} \delta\left\{\Phi_{f s i}\right\}\right) d t \\
& =-\int_{t_{1}}^{t_{2}}\left(\rho A_{f_{s i}}\left\{\dot{u}_{f s i}\right\} \delta\left\{\Phi_{f s i}\right\}\right) d t
\end{aligned}
$$

A further simplification can be obtained by defining a fluid-structure interaction matrix $\Omega$ such that

$$
\begin{aligned}
& \Omega_{i j}=\rho A_{f s i} \text { for structure node } \mathrm{i} \text { connected to fluid node } \mathrm{j} \\
& \Omega=0 \quad \text { Otherwise }
\end{aligned}
$$

This definition for $\Omega$ automatically accounts for the particular nodes that are involved in the fluid-structure interaction. The equation for II can now be written as

$$
I I=\int_{t_{1}}^{t_{2}}\left(-[\Omega]^{T}\{\dot{u}\} \delta\{\Phi\}\right) d t
$$

Substituting for $I, I I$, and $\Omega$ yields

$$
\begin{gathered}
\int_{t_{1}}^{t_{2}}\left(-\left(\left[M_{s}\right]\{\ddot{u}\}+\left[K_{s}\right]\{u\}\right) \delta\{u\}+\left(\left[M_{f}\right]\{\ddot{\Phi}\}+\left[K_{f}\right]\{\Phi\}\right) \delta\{\Phi\}\right) d t \\
+\int_{t_{1}}^{t_{2}}\left(\left(F_{s}-C_{s}\{\dot{u}\}-\Omega\{\dot{\Phi}\}\right) \delta\{u\}-\Omega^{T}\{\dot{u}\} \delta\{\Phi\}\right) d t=0
\end{gathered}
$$

Rearranging terms, we have

$$
\begin{gathered}
\int_{t_{1}}^{t_{2}}\left(-\left(\left[M_{s}\right]\{\ddot{u}\}+\left[C_{s}\right]\{\dot{u}\}+\left[K_{s}\right]\{u\}-F_{s}+\Omega\{\dot{\Phi}\}\right) \delta\{u\}\right) d t \\
+\int_{t_{1}}^{t_{2}}\left(\left(\left[M_{f}\right]\{\ddot{\Phi}\}+\left[K_{f}\right]\{\Phi\}-\Omega^{T}\{\dot{u}\}\right) \delta\{\Phi\}\right) d t=0
\end{gathered}
$$


Since the equation must hold for any arbitrary value of the virtual displacements $\delta\{u\}$ and $\delta\{\Phi\}$, each integrand must equal zero.

and

$$
\begin{gathered}
{\left[M_{s}\right]\{\ddot{u}\}+\left[C_{s}\right]\{\dot{u}\}+\left[K_{s}\right]\{u\}+\Omega\{\dot{\Phi}\}=F_{s},} \\
-\left[M_{f}\right]\{\ddot{\Phi}\}-\left[K_{f}\right]\{\Phi\}+\Omega^{T}\{\dot{u}\}=0
\end{gathered}
$$

Arranging the equations in a matrix form and summing over all of the elements to form the global matrices gives

$$
\left[\begin{array}{cc}
M_{s} & 0 \\
0 & -M_{f}
\end{array}\right]\left\{\begin{array}{l}
\ddot{u} \\
\ddot{\Phi}
\end{array}\right\}+\left[\begin{array}{cc}
C_{s} & \Omega \\
\Omega^{T} & 0
\end{array}\right]\left\{\begin{array}{l}
\dot{u} \\
\dot{\Phi}
\end{array}\right\}+\left[\begin{array}{cc}
K_{s} & 0 \\
0 & -K_{f}
\end{array}\right]\left\{\begin{array}{l}
u \\
\Phi
\end{array}\right\}=\left\{\begin{array}{l}
F \\
0
\end{array}\right\}
$$

where the element fluid matrices are given in equations 18 and 20, and the fluidstructure coupling matrix $\Omega$ is given by equation 27 . The matrices in equation 33 are global matrices formed by summing over the entire finite element domain.

If the actuator dynamics are not of interest, equation 33 can be simplified to

$$
\left[M_{f}\right]\{\ddot{\Phi}\}+\left[K_{f}\right]\{\Phi\}=\Omega^{T}\{\dot{u}\}
$$

where the structure has an imposed velocity $\dot{u}$. This form of the equation is particularly useful in evaluating the response for the entire resonator drive scheme of Lucas. In this case, equation 34 is used along with the entire internal surface area of the resonator in the axial direction as the interface area in the coupling matrix. 


\subsection{Piezoelectric Actuator models}

The simple spring/mass/damper system will now be expanded to a more realistic actuator for the acoustic cavity. Two types of piezoelectric actuators were considered in this study, a stacked actuator, and a bimorph actuator. In the stacked type of actuator, piezoelectric layers are arranged in such a way that their deflections add together when voltage is applied across the stack. Each layer is actuated in its 3-3 mode, which means they are intended to expand axially. A bimorph actuator expands in the radial direction (1-3 mode), but the two layers are arranged such that as one expands, the other contracts. This causes the disc to bend. Figure 7 shows the standard notation defining directions in a piezoelectric material. A cross section of the two types of actuators considered is shown in Figure 8. The letter $\mathrm{P}$ and an arrow indicate the poling direction of the material.

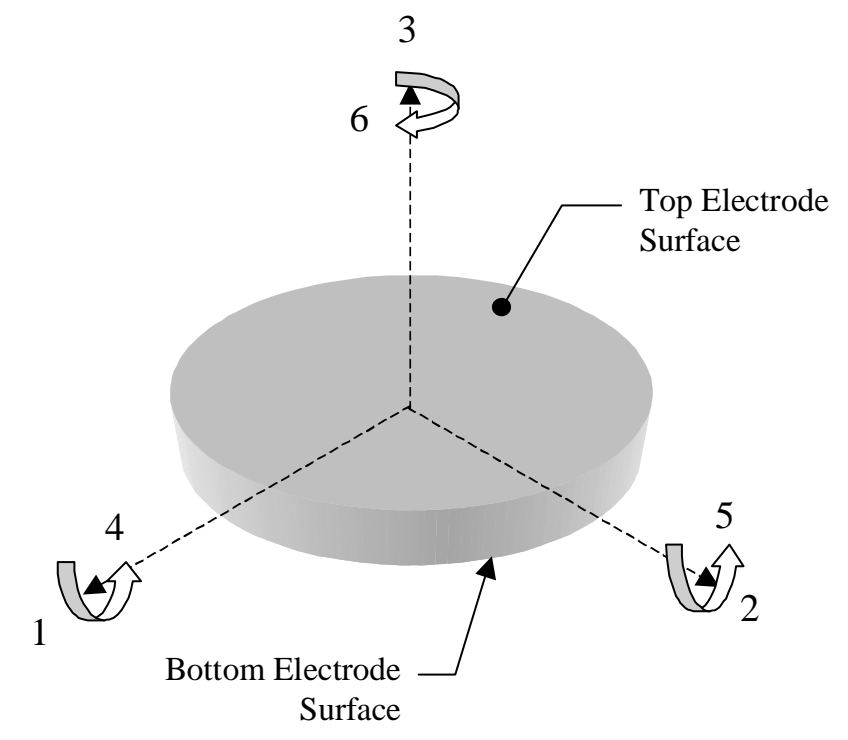

Figure 7 - Standard Directions in Piezoelectric Material 


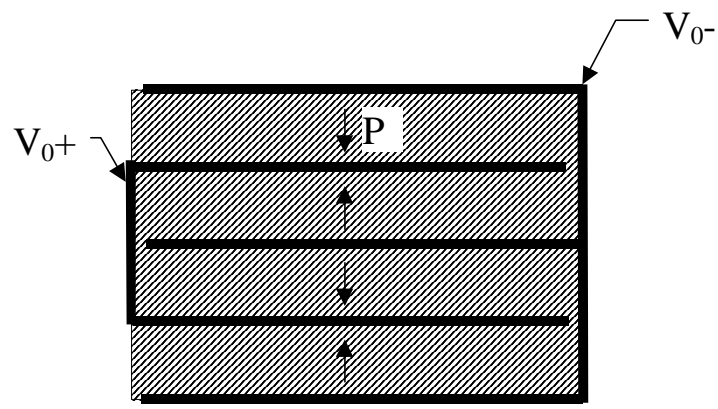

Cross Section of Stacked Type Actuator

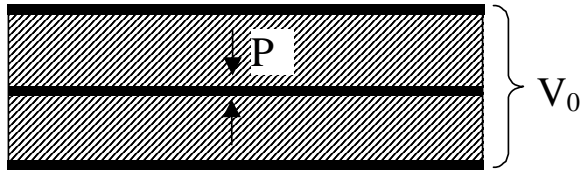

Cross Section of Bimorph Type Actuator

Figure 8 - Typical Piezoelectric Actuator Types

\subsubsection{General Piezoelectric Modeling}

The Piezoelectric materials will be modeled based on the linear theory of piezoelectricity as defined in IEEE Standard 176. One form of the constitutive equations is

and

$$
\begin{gathered}
T_{p}=c_{p q}^{E} S_{q}-e_{j p} E_{j}, \\
D_{p}=e_{p q} S_{q}+\varepsilon_{j p} E_{j}
\end{gathered}
$$

or in matrix form:

$$
\left\{\begin{array}{l}
T \\
D
\end{array}\right\}=\left[\begin{array}{cc}
c^{E} & -e \\
e^{T} & \varepsilon
\end{array}\right]\left\{\begin{array}{l}
S \\
E
\end{array}\right\}
$$

where $T=$ mechanical stress, $c^{E}=$ compliance matrix at constant electric field, $S=$ mechanical strain, $e=$ piezoelectric constant matrix, $D=$ electric displacement, $\varepsilon=$ permittivity matrix at constant stress, and $E=$ electric field. This form for the equations is the same as that used by ANSYS. This will allow the use of the same material properties when comparing the model created here and the commercial code. 
We will use the Lagrange equations to create the finite element formulation

$$
\frac{d}{d t}\left(\frac{\partial T_{K E}}{\partial \dot{q}}\right)-\frac{\partial T_{K E}}{\partial q}+\frac{\partial U}{\partial q}=Q_{n c}
$$

where $T_{K E}$ is the kinetic energy, $U$ is the potential energy, $q$ is the generalized coordinate, and $Q_{n c}$ is the non-conservative force. The subscript $K E$ is added to the kinetic energy to avoid confusion with the mechanical stress. The energy expressions given by Baz (2001) are

$$
\begin{gathered}
U=\frac{1}{2} \int_{V} S^{T} T d V+\frac{1}{2} \int_{V} D^{T} E d V \\
T_{K E}=\frac{1}{2} \int_{V} \rho \dot{u}^{2} d V
\end{gathered}
$$

The finite element model will be based on the mechanical and electrical displacements at the nodes of the elements. This formulation will simplify the application of an electrical potential across the piezoelectric as a boundary condition. Therefore, the stresses and electric field will need to be eliminated from equation 38. Solving the second of equations 36 for $E$ and substituting in the first equation yields

$$
\{E\}=-[\varepsilon]^{-1}[e]^{T}\{S\}+[\varepsilon]^{-1}[D]
$$

and

$$
\{T\}=\left(\left[c^{E}\right]+[e][\varepsilon]^{-1}[e]^{T}\right)\{S\}-[e][\varepsilon]^{-1}\{D\}
$$

\subsubsection{Potential Energy}

Substituting equations 40 into the potential energy equation yields 


$$
\begin{aligned}
U & =\underbrace{\frac{1}{2}\left\{\{S\}_{V}^{T}\left(\left[c^{E}\right]+[e][\varepsilon]^{-1}[e]^{T}\right)\{S\} d V\right.}_{I}-\underbrace{\frac{1}{2} \int_{V}\{S\}^{T}[e][\varepsilon]^{-1}\{D\} d V}_{I I} \\
& -\underbrace{\frac{1}{2} \int_{V}\{D\}^{T}[\varepsilon]^{-1}[e]^{T}\{S\} d V}_{I I I}+\underbrace{\frac{1}{2} \int_{V}\{D\}^{T}[\varepsilon]^{-1}\{D\} d V}_{I V}
\end{aligned}
$$

In the finite element formulation, the mechanical and electric displacements will be approximated by shape functions as follows

$$
\{u\}=\left[N_{u}\right]\left\{\Delta_{u}\right\},
$$

and

$$
\{D\}=\left[N_{d}\right]\left\{\Delta_{d}\right\}
$$

where $\{\Delta\}$ is the appropriate nodal deflection vector. The mechanical strain can be related to the mechanical displacement by applying the appropriate operation on the shape function. This gives the strain as

$$
\{S\}=\left[B_{u}\right]\left\{\Delta_{u}\right\}
$$

The $\left[B_{u}\right]$ matrix is a function of the $\left[N_{u}\right]$ matrix and depends on the specific geometry being modeled. With these substitutions, and noting that $I I I$ is the transpose of $I I$, the pieces of equation 41 become

and

$$
\begin{gathered}
I=\frac{1}{2} \int_{V}\left\{\Delta_{u}\right\}^{T}\left\{B_{u}\right\}^{T}\left(\left[c^{E}\right]+[e][\varepsilon]^{-1}[e]^{T}\right)\left\{B_{u}\right\}\left\{\Delta_{u}\right\} d V, \\
I I=-\frac{1}{2} \int_{V}\left\{\Delta_{u}\right\}^{T}\left\{B_{u}\right\}^{T}[e][\varepsilon]^{-1}\left\{N_{d}\right\}\left\{\Delta_{d}\right\} d V, \\
\left.I V=\frac{1}{2} \int_{V}\left\{\Delta_{d}\right\}^{T}\left\{N_{d}\right\}^{T}[\varepsilon]^{-1}\left\{N_{d}\right\} \Delta_{d}\right\} d V
\end{gathered}
$$

The potential energy may now be written as

$$
\begin{aligned}
U & =\frac{1}{2}\left\{\Delta_{u}\right\}^{T}\left[K_{u u}\right]\left\{\Delta_{u}\right\}-\frac{1}{2}\left\{\Delta_{u}\right\}^{T}\left[K_{u d}\right]\left\{\Delta_{d}\right\} \\
& \left.\left.-\frac{1}{2}\left\{\Delta_{d}\right\}^{T}\left[K_{d u}\right]\right\} \Delta_{u}\right\}+\frac{1}{2}\left\{\Delta_{d}\right\}^{T}\left[K_{d d}\right]\left\{\Delta_{d}\right\}
\end{aligned}
$$


where

$$
\begin{aligned}
& K_{u u}=\int_{V}\left\{B_{u}\right\}^{T}\left(\left[c^{E}\right]+[e][\varepsilon]^{-1}[e]^{T}\right)\left\{B_{u}\right\} d V \\
& K_{u d}=\int_{V}\left\{B_{u}\right\}^{T}[e][\varepsilon]^{-1}\left\{N_{d}\right\} d V \\
& K_{d u}=K_{u d}{ }^{T} \\
& K_{d d}=\int_{V}\left\{N_{d}\right\}^{T}[\varepsilon]^{-1}\left\{N_{d}\right\} d V
\end{aligned}
$$

A final simplification can be made by defining

$$
\begin{gathered}
K=\left[\begin{array}{cc}
K_{u u} & -K_{u d} \\
-K_{u d}^{T} & K_{d d}
\end{array}\right], \\
\{\Delta\}=\left\{\begin{array}{l}
\Delta_{u} \\
\Delta_{d}
\end{array}\right\}
\end{gathered}
$$

and

then the potential energy is

$$
U=\frac{1}{2}\{\Delta\}^{T}[K][\Delta\}
$$

\subsubsection{Kinetic Energy}

The kinetic energy is easily written by substituting the definition for the mechanical displacement from equation 42 into equation 39.

$$
T_{K E}=\frac{1}{2} \int_{V} \rho\left\{\dot{\Delta}_{u}\right\}^{T}\left[N_{u}\right]^{T}\left[N_{u}\right]\left\{\dot{\Delta}_{u}\right\} d V
$$

which is written more compactly as

$$
T_{K E}=\frac{1}{2}\{\dot{\Delta}\}^{T}[M]\{\dot{\Delta}\}
$$


where

$$
M=\left[\begin{array}{cc}
M_{u u} & 0 \\
0 & 0
\end{array}\right], M_{u и}=\int_{V} \rho\left[N_{u}\right]^{T}\left[N_{u}\right] d V,
$$

and

$$
\{\dot{\Delta}\}=\left\{\begin{array}{l}
\dot{\Delta}_{u} \\
\dot{\Delta}_{d}
\end{array}\right\}
$$

\subsubsection{Non-conservative force}

The non-conservative forces can be determined from the virtual work

$$
\delta W=Q_{n c} \delta\{q\}
$$

For the piezoelectric device used as an actuator, the exciting force is the applied electrical potential. The virtual work will be

$$
\delta W=\delta Q V_{0}
$$

where $Q$ is the surface charge and $V_{0}$ is the applied voltage. The charge on the piezoelectric material layers is given by

$$
Q=\oint_{s} D d s
$$

where $s$ is the surface area. Substituting the finite element formulation for $D$ we have

$$
Q=\oint_{s}\left[N_{d}\right]\left\{\Delta_{d}\right\} d s
$$

The virtual work is thus

$$
\delta W=\oint_{s} V_{0}\left[N_{d}\right] d s\left\{\delta \Delta_{d}\right\}
$$

By inspection, we see that the non-conservative force is

$$
Q_{n c}=\oint_{s} V_{0}\left[N_{d}\right] d s
$$




\subsubsection{Equations of motion}

It is now a simple substitution of the preceding into the Lagrange equation to obtain the equations of motion

$$
[M]\{\ddot{\Delta}\}+[K]\{\Delta\}=\left(Q_{n c}\right)
$$

In an expanded form, the above equation is written as

$$
\left[\begin{array}{cc}
M_{u и} & 0 \\
0 & 0
\end{array}\right]\left\{\begin{array}{l}
\ddot{\Delta}_{u} \\
\ddot{\Delta}_{d}
\end{array}\right\}+\left[\begin{array}{cc}
K_{u u} & -K_{u d} \\
-K_{u d}^{T} & K_{d d}
\end{array}\right]\left\{\begin{array}{l}
\Delta_{u} \\
\Delta_{d}
\end{array}\right\}=\left(\begin{array}{c}
0 \\
Q_{n c}
\end{array}\right)
$$

Combining the piezoelectric actuator with the fluid matrices gives

$$
\left[\begin{array}{ccc}
M_{u u} & 0 & 0 \\
0 & 0 & 0 \\
0 & 0 & -M_{f}
\end{array}\right]\left\{\begin{array}{l}
\ddot{\Delta}_{u} \\
\ddot{\Delta}_{d} \\
\ddot{\Phi}
\end{array}\right\}+\left[\begin{array}{ccc}
C_{u u} & 0 & \Omega \\
0 & 0 & 0 \\
\Omega^{T} & 0 & 0
\end{array}\right]\left\{\begin{array}{c}
\dot{\Delta}_{u} \\
\dot{\Delta}_{d} \\
\dot{\Phi}
\end{array}\right\}+\left[\begin{array}{ccc}
K_{u u} & -K_{u d} & 0 \\
-K_{u d}^{T} & K_{d d} & 0 \\
0 & 0 & -K_{f}
\end{array}\right]\left\{\begin{array}{c}
\Delta_{u} \\
\Delta_{d} \\
\Phi
\end{array}\right\}=\left\{\begin{array}{c}
0 \\
Q_{n c} \\
0
\end{array}\right\}(60)
$$

\subsubsection{Drive Power}

The actuator will require an electrical drive unit to supply voltage and current. The sizing of this power supply depends on knowing the required power output. The electrical power is given by

$$
P=I V_{0}
$$

where $P=$ electrical power, $I=$ current, and $V_{0}=$ voltage. For harmonic drive at a frequency $\omega$,

$$
I=\frac{d Q}{d t}=i \omega Q
$$




$$
P=V_{0} i \omega \oint_{s}\left[N_{d}\right]\left\{\Delta_{d}\right\} d s
$$

Comparing this expression with the non-conservative force in equation 57 we see that

$$
P=i \omega Q_{n c}\left\{\Delta_{d}\right\}
$$

For sinusoidal voltage input, the RMS power is simply

$$
P_{R M S}=\frac{\sqrt{2}}{2} P
$$

Once the nodal displacements are calculated, the power is found by applying equation 64 or 65 .

\subsubsection{Derivation of FE model of stacked actuator}

A single layer finite element model of a stacked actuator is shown in Figure 9 below. A local coordinate $x$ is defined which varies from 0 at the leftmost node $i$ to $\mathrm{L}$ at the rightmost node $k$. Comparing this figure with Figure 7, we see that the $x$ coordinate corresponds to the standard piezoelectric direction 3. 


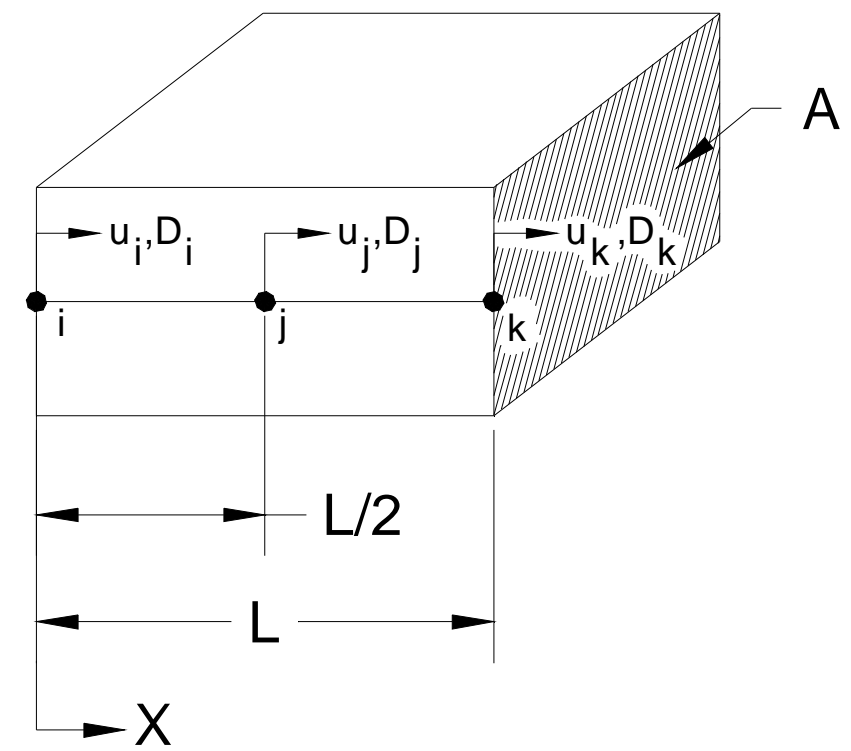

Figure 9 - Three Noded Stacked Actuator Finite Element

The mechanical displacement is assumed to vary quadratically along the element. The interpolation equation is the same as that used for the velocity potential. Substituting $u$ for $\phi$ in equation 14 gives

$$
u(x)=\left\{1-3 \frac{x}{L}+2\left(\frac{x}{L}\right)^{2} 4 \frac{x}{L}-4\left(\frac{x}{L}\right)^{2}-\frac{x}{L}+2\left(\frac{x}{L}\right)^{2}\right\}\left\{\begin{array}{l}
u_{i} \\
u_{j} \\
u_{k}
\end{array}\right\}
$$

Writing this equation in the same form as the general derivation given in section 2.2.1 gives

$$
\left.u(x)=[N]\} \Delta_{u}\right\}
$$

Noting that $S=\frac{d u}{d x}=u_{x}$, the $B_{u}$ matrix is

$$
B_{u}=\left[N_{x}\right]
$$


where the subscript in the interpolation matrix denotes differentiation with respect to $x$. As $\nabla D=0$, we must have $D_{i}=D_{j}=D_{k}$. This means the interpolation function for $D$ is the identity function. Denoting the cross sectional area by $A$ and substituting these definitions into the equations for the stiffness matrices yields

$$
\begin{gathered}
K_{u u}=A \int_{0}^{L}\left\{N_{x}\right\}^{T}\left(\left[c^{E}\right]+[e][\varepsilon]^{-1}[e]^{T}\right)\left\{N_{x}\right\} d x, \\
K_{u d}=A \int_{0}^{L}\left\{N_{x}\right\}^{T}[e][\varepsilon]^{-1}\{1\} d x, \\
K_{d d}=A \int_{0}^{L}[\varepsilon]^{-1} d x=A L[\varepsilon]^{-1}
\end{gathered}
$$

and

The mass matrix is given by

$$
\left[M_{u u}\right]=\int_{0}^{L} \rho A\{N\} d x
$$

The non-conservative electrical force is

$$
Q_{n c}=V_{0} A
$$

The element deflection vector $\Delta$ is

$$
\Delta^{T}=\left\{\begin{array}{llll}
u_{\mathrm{i}} & \mathrm{u}_{\mathrm{j}} & \mathrm{u}_{\mathrm{k}} & \mathrm{D}_{\mathrm{i}}
\end{array}\right\}
$$

Substituting the preceding expressions into the equations of motion

$$
\left[\begin{array}{cc}
M_{u и} & 0 \\
0 & 0
\end{array}\right]\{\ddot{\Delta}\}+\left[\begin{array}{cc}
K_{u u} & -K_{u d} \\
-K_{u d}^{T} & K_{d d}
\end{array}\right]\{\Delta\}=\left(\begin{array}{c}
0_{3 x 1} \\
V_{0} A
\end{array}\right)
$$

Equation 75 along with equations $69,70,71$, and 72 form the finite element description for a single layer of the actuator. These layers are then stacked up to get the desired deflection. This leads to the drawback of using this type of actuator. The first order performance of this type of actuator from Near (1996) is 


$$
\Delta L=n d_{33} V_{0},
$$

and

$$
\text { Power }=2 \pi f C V_{0}^{2}(1+\tan \delta)
$$

where $\Delta L$ is the change in length, $n$ is the number of layers, $f$ is the actuation frequency, $\tan \delta$ is the loss tangent, and $C$ is the capacitance

$$
C=n \varepsilon^{T} A / t
$$

For a typical material such as $\mathrm{PZT}-5 \mathrm{H}$, the material properties are

$$
\begin{array}{ll}
d_{33}=595 \times 10^{-12} \mathrm{~m} / \text { Volt } & \varepsilon^{T}{ }_{33}=3400 \varepsilon_{0} \\
t=0.125 \mathrm{~mm} & \varepsilon_{0}=8.8542 \times 10^{-12} \mathrm{Farad} / \mathrm{m} \\
V_{\max }=120 \text { Volt } &
\end{array}
$$

Given these parameters, each layer contributes only $0.149 \mu \mathrm{m}$ to the deflection. For a target deflection of $40 \mu \mathrm{m}, 560$ layers are required. The power required to drive a 560 layer, two-inch diameter actuator at $100 \mathrm{~Hz}$ would be approximately 2500 watts. A more efficient design could possibly be obtained by using the bimorph, as it only has two layers contributing to the total capacitance. The derivation of the finite element model for the bimorph actuator follows.

\subsubsection{Derivation of FE model for axisymmetric bimorph disc}

Before jumping into the FE formulas, we will first make some simplifying assumptions regarding what components of stress and strain can be expected.

Dobrucki and Pruchnicki (1997) present a finite element theory for vibrations of a piezoelectric bimorph. Unfortunately, they assume that since the stress in the $\mathrm{z}$-axis is 
negligible (Kirchoff hypothesis), the strain may also be ignored. We will see that this is not the case for piezoelectric materials.

Referring back to the constitutive equations,

and

$$
\begin{gathered}
T_{p}=c_{p q}^{E} S_{q}-e_{j p} E_{j}, \\
D_{p}=e_{p q} S_{q}+\varepsilon_{j p} E_{j}
\end{gathered}
$$

For axisymmetric vibrations, $p$ and $q=1-3$ refer to the radial (1), angular (2), and vertical (3) directions, as in Figure 10. The arrows indicate the poling direction of the material. Actuation voltage is applied in the 3 direction; therefore, $j$ is set to 3 .

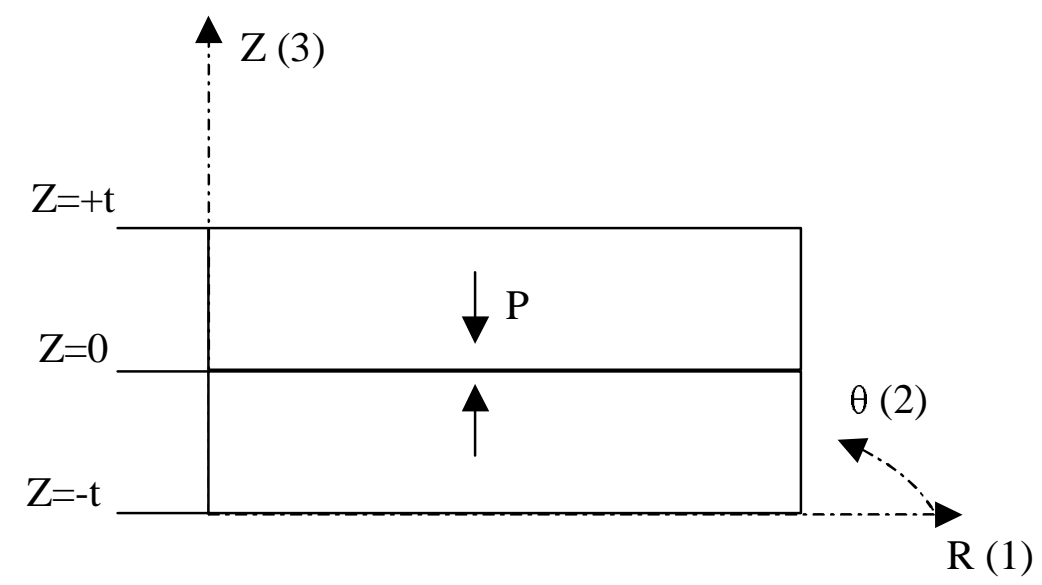

Figure 10 - Cross Section of Bimorph

Noting that the material properties in direction 1 equal those in direction 2, we have for the stress equations

and

$$
\begin{aligned}
& T_{1}=c_{11}^{E} S_{1}+c_{12}^{E} S_{2}+c_{13}^{E} S_{3}-e_{31} E_{3}, \\
& T_{2}=c_{12}^{E} S_{1}+c_{22}^{E} S_{2}+c_{13}^{E} S_{3}-e_{31} E_{3}, \\
& T_{3}=c_{31}^{E} S_{1}+c_{31}^{E} S_{2}+c_{33}^{E} S_{3}-e_{33} E_{3}
\end{aligned}
$$


For flexural vibrations of a thin disc, it is reasonable to make the Kirchoff assumption that $T_{3}=0$. The $T_{3}$ equation can therefore be solved for $S_{3}$,

or

$$
\begin{gathered}
c_{31}^{E}\left(S_{1}+S_{2}\right)+c_{33}^{E} S_{3}-e_{33} E_{3}=0, \\
S_{3}=\frac{e_{33}}{c_{33}^{E}} E_{3}-\frac{c_{31}^{E}}{c_{33}^{E}}\left(S_{1}+S_{2}\right)
\end{gathered}
$$

Substituting this expression for $S_{3}$ into the equations for $T_{1}$ and $T_{2}$, and noting that matrix $c^{E}$ is symmetric, we have

or

$$
\begin{gathered}
T_{1}=c_{11}^{E} S_{1}+c_{12}^{E} S_{2}+c_{13}^{E}\left(\frac{e_{33}}{c_{33}^{E}} E_{3}-\frac{c_{31}^{E}}{c_{33}^{E}} S_{1}-\frac{c_{31}^{E}}{c_{33}^{E}} S_{2}\right)-e_{31} E_{3}, \\
T_{1}=\left(c_{11}^{E}-\frac{\left(c_{13}^{E}\right)^{2}}{c_{33}^{E}}\right) S_{1}+\left(c_{12}^{E}-\frac{\left(c_{13}^{E}\right)^{2}}{c_{33}^{E}}\right) S_{2}-\left(e_{31}-\frac{c_{13}^{E}}{c_{33}^{E}} e_{33}\right) E_{3}
\end{gathered}
$$

giving

$$
T_{1}=c_{11}^{E^{*}} S_{1}+c_{12}^{E^{*}} S_{2}-e_{31}^{*} E_{3}
$$

where we have defined effective compliance and piezoelectric constants as in Ling-Hui (1998)

$$
\begin{gathered}
c_{11}^{E^{*}} \equiv c_{11}^{E}-\frac{\left(c_{13}^{E}\right)^{2}}{c_{33}^{E}}, \\
c_{12}^{E^{*}} \equiv c_{12}^{E}-\frac{\left(c_{13}^{E}\right)^{2}}{c_{33}^{E}}, \\
e_{31}^{*} \equiv e_{31}-\frac{c_{13}^{E}}{c_{33}^{E}} e_{33}
\end{gathered}
$$

and

Similarly,

$$
T_{2}=c_{12}^{E^{*}} S_{1}+c_{11}^{E^{*}} S_{2}-e_{31}^{*} E_{3}
$$

Thus, the stress equations are reduced to two equations with the equivalent compliance and piezoelectric constants as defined above. 
The actuation voltage and electrodes are in the 3 direction, giving rise to an electric displacement also in the 3 direction

$$
D_{3}=e_{31} S_{1}+e_{31} S_{2}+e_{33} S_{3}+\varepsilon_{33} E_{3}
$$

Substituting the expression for $S_{3}$ from above

and simplifying

$$
D_{3}=e_{31} S_{1}+e_{31} S_{2}+e_{33}\left[\frac{e_{33}}{c_{33}^{E}} E_{3}-\frac{c_{31}^{E}}{c_{33}^{E}}\left(S_{1}+S_{2}\right)\right]+\varepsilon_{33} E_{3}
$$

where the effective permittivity is defined as

$$
\varepsilon_{33}^{*} \equiv \varepsilon_{33}+\frac{e_{33}^{2}}{c_{33}}
$$

In summary, there are two components to the stress and strain matrix, and one component to the electric displacement and electric field matrices. The constitutive matrices for this type of actuator are reduced to

$$
\begin{gathered}
\{T\}=\left\{\begin{array}{l}
T_{1} \\
T_{2}
\end{array}\right\},\{S\}=\left\{\begin{array}{l}
S_{1} \\
S_{2}
\end{array}\right\},\{D\}=\left\{D_{3}\right\},\{E\}=\left\{E_{3}\right\} \\
{\left[c^{E}\right]=\left[\begin{array}{ll}
c_{11}^{E^{*}} & c_{12}^{E^{*}} \\
c_{12}^{E^{*}} & c_{11}^{E^{*}}
\end{array}\right],[e]=\left[\begin{array}{c}
e_{31}^{*} \\
e_{31}^{*}
\end{array}\right],[\varepsilon]=\left[\varepsilon_{33}^{*}\right]}
\end{gathered}
$$

The relationship between the displacements and the strain are needed in order to write the finite element matrices. Let the bimorph be divided into finite elements of length $L$ as shown in Figure 11. There is no deformation of the element along the central axis, and planes normal to the axis are assumed to remain normal after deformation. The 
dashed lines indicate the undeformed shape of the element. The inner radius node of the element is designated node $i$ and the outer radius node as $j$. A coordinate $x$ is introduced which varies from 0 at node $i$ to $\mathrm{L}$ at node $j$. The radius of a location along the element is

$$
r(x)=r_{i}+x
$$

where $r_{i}$ refers to the radius of node $i$. The coordinate $z$ is taken along the thickness of the element and varies from $-t$ to $+t$.

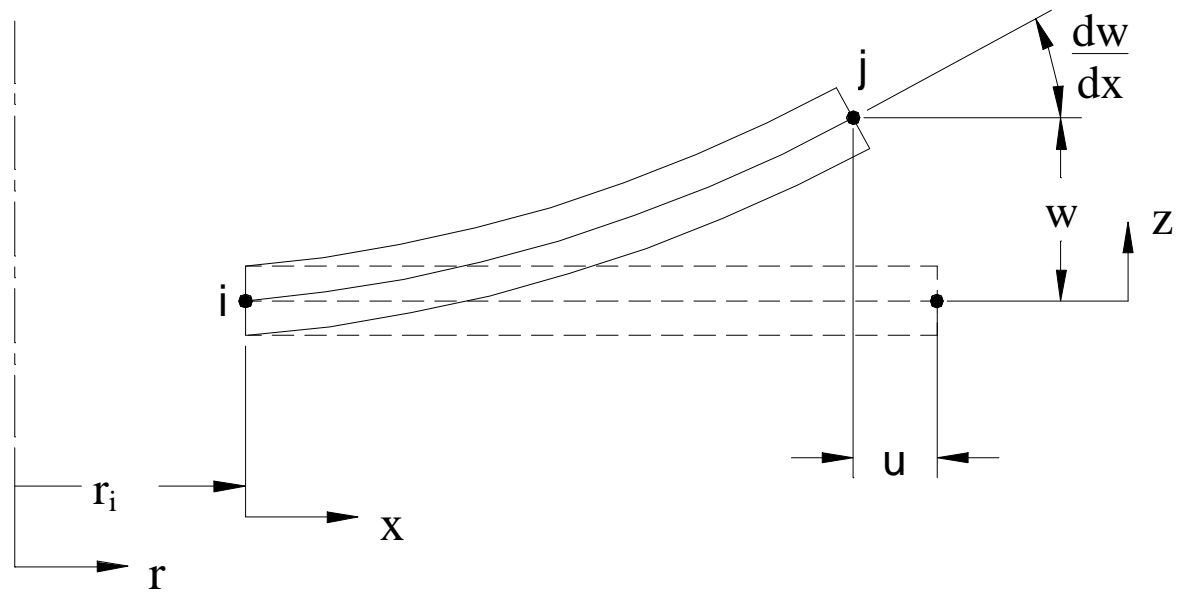

Figure 11 - Typical Axisymmetric Bimorph Finite Element

From Figure 11, the radial displacement $u$ of any point through the thickness of the actuator is

$$
u=z \frac{d w}{d x}
$$

The radial strain is therefore 


$$
S_{1}=\frac{\partial u}{\partial x}=z \frac{d^{2} w}{d x^{2}}
$$

The circumferential or hoop strain is

$$
S_{2}=\frac{(r+u) d \theta-r d \theta}{r d \theta}=\frac{z}{r} \frac{d w}{d x}
$$

Let $w(x)$ be described by a cubic equation as follows

$$
w(x)=a_{1}+a_{2} x+a_{3} x^{2}+a_{4} x^{3}
$$

or, in matrix form

$$
w(x)=\left(\begin{array}{llll}
1 & x & x^{2} & x^{3}
\end{array}\right)\left(\begin{array}{llll}
a_{1} & a_{2} & a_{3} & a_{4}
\end{array}\right)^{T}
$$

The boundary conditions are

$$
w(0)=w_{i}, w(L)=w_{j}, w_{x}(0)=\theta_{i}, w_{x}(L)=\theta_{j}
$$

where $\theta=w_{x}$. The boundary conditions in terms of the nodal deflections are

and

$$
\begin{gathered}
w(0)=a_{1}=w_{i}, \\
w(L)=a_{1}+a_{2} L+a_{3} L^{2}+a_{4} L^{3}=w_{j}, \\
\theta(0)=a_{2}=\theta_{i}, \\
\theta(L)=a_{2}+2 a_{3} L+3 a_{4} L^{2}=\theta_{j}
\end{gathered}
$$

Following a similar procedure to that given in the fluid modeling section, the out of plane deflection in terms of the nodal deflection values is

$$
w(x)=\left[N_{u}\right]\left(\Delta_{u}\right)
$$

where $\left[N_{u}\right]=\left[1-3\left(\frac{x}{L}\right)^{2}+2\left(\frac{x}{L}\right)^{3} x\left(1-2\left(\frac{x}{L}\right)+\left(\frac{x}{L}\right)^{2}\right) 3\left(\frac{x}{L}\right)^{2}-2\left(\frac{x}{L}\right)^{3} x\left(-\left(\frac{x}{L}\right)+\left(\frac{x}{L}\right)^{2}\right)\right]$,

and

$$
\left(\Delta_{u}\right)=\left(\begin{array}{llll}
w_{i} & \theta_{i} & w_{j} & \theta_{j}
\end{array}\right)^{T}
$$

Substituting $w$ into equations 92 and 93 yields the $B_{u}$ matrix 


$$
B_{u}=\left[\begin{array}{c}
z N_{x x} \\
\frac{z}{r_{i}+x} N_{x}
\end{array}\right]=z\left[\begin{array}{c}
N_{x x} \\
\frac{1}{r_{i}+x} N_{x}
\end{array}\right]
$$

For the electric displacement, a simple linear interpolation function is used

$$
\begin{aligned}
D(x)=\left[\begin{array}{ll}
1-\frac{x}{L} & \frac{x}{L}
\end{array}\right]\left(\begin{array}{c}
D_{i} \\
D_{j}
\end{array}\right) \\
\text { or } \quad D(x)=\left[N_{d}\right]\left(\Delta_{d}\right)
\end{aligned}
$$

The preceding can now be substituted into the mass and stiffness matrix equations. For an axisymmetric element, the differential volume element is $d V=2 \pi r d z d x$

$$
\begin{aligned}
K_{u u} & =\int_{V}\left[N_{x x}{ }^{T} \frac{1}{r_{i}+x} N_{x}^{T}\right] z^{2}\left(\left[c^{E}\right]+[e][\varepsilon]^{-1}[e]^{T}\right)\left[\begin{array}{c}
N_{x x} \\
\frac{1}{r_{i}+x}
\end{array} N_{x}\right] d V \\
& =2 \pi \int_{0}^{L}\left[N_{x x}{ }^{T} \frac{1}{r_{i}+x} N_{x}^{T}\right]\left\{\int_{-t}^{+t} z^{2}\left(\left[c^{E}\right]+[e][\varepsilon]^{-1}[e]^{T}\right) d z\right\}\left[\begin{array}{c}
N_{x x} \\
\frac{1}{r_{i}+x} N_{x}
\end{array}\right]\left(r_{i}+x\right) d x
\end{aligned}
$$

The integral in curly braces above can be simplified as

$$
\begin{aligned}
{[\mathscr{Q}]_{e} } & =\int_{-t}^{+t} z^{2}\left(\left[c^{E}\right]+[e][\varepsilon]^{-1}[e]^{T}\right) d z \\
& =\frac{2}{3} t^{3}\left(\left[c^{E}\right]+[e][\varepsilon]^{-1}[e]^{T}\right)
\end{aligned}
$$

The electro-mechanical coupling matrix is

$$
\begin{aligned}
K_{u d} & =\int_{V}\left[\begin{array}{ll}
N_{x x}{ }^{T} & \frac{1}{r_{i}+x} N_{x}{ }^{T}
\end{array}\right] z[e][\varepsilon]^{-1}\left\{N_{d}\right\} d V \\
& =2 \pi \int_{0}^{L}\left[N_{x x}{ }^{T} \frac{1}{r_{i}+x} N_{x}^{T}\right]\left\{\int_{-t}^{+t} z[e][\varepsilon]^{-1} d z\right\}\left\{N_{d}\right\}\left(r_{i}+x\right) d x
\end{aligned}
$$

For a bimorph, the top and bottom layer piezoelectric matrices have equal magnitude but opposite signs. Considering the integral in curly braces we have 


$$
\begin{aligned}
\int_{-t}^{+t} z[e][\varepsilon]^{-1} d z & =\int_{-t}^{0} z[-e][\varepsilon]^{-1} d z+\int_{0}^{t} z[e][\varepsilon]^{-1} d z \\
& =t^{2}[e][\varepsilon]^{-1}
\end{aligned}
$$

then,

$$
K_{u d}=2 \pi t^{2}[e][\varepsilon]^{-1} \int_{0}^{L}\left[\begin{array}{ll}
N_{x x}{ }^{T} & \frac{1}{r_{i}+x} N_{x}{ }^{T}
\end{array}\right]\left\{N_{d}\right\}\left(r_{i}+x\right) d x
$$

The electric displacement stiffness matrix reduces to

$$
K_{d d}=2 \pi 2 t[\varepsilon]^{-1} \int_{0}^{L}\left\{N_{d}\right\}^{T}\left\{N_{d}\right\}\left(r_{i}+x\right) d x
$$

The mechanical mass matrix is written as

$$
M_{u u}=2 \pi 2 t \rho \int_{0}^{L}\left[N_{u}\right]^{T}\left[N_{u}\right]\left(r_{i}+x\right) d x
$$

The final piece required to fully describe the piezoelectric bimorph is the nonconservative force

$$
Q_{n c}=\oint_{s} V_{0}\left[N_{d}\right] d s
$$

There are three surfaces to consider in this integral, the top electrode, bottom electrode, and the cylindrical side of the piezoelectric bimorph. Since $D_{3}$ is parallel to the sides, this contribution to the charge will be zero. We will also consider either the top or the bottom electrode to be grounded as a boundary condition. This leaves only the integral over one of the electrodes, choose the upper electrode surface for convenience. At this position, $z=t, d s=r d \theta d r=2 \pi\left(r_{i}+x\right) d x$

$$
Q_{n c}=2 \pi V_{0} \int_{0}^{L}\left[N_{d}\right]^{T}\left(r_{i}+x\right) d x
$$

where the transpose has been taken to make the force consistent with the vector scheme used. The voltage $V_{0}$ refers to the peak amplitude across both layers of the bimorph. 
Each bimorph element is assembled into the global matrix. This global matrix is then inserted into the fluid-structure interaction equations. The bimorph actuator is discretized along the radial direction, while the fluid is discretized along the axial direction. The fluid-structure interaction matrix allows the multiple structural nodes to connect with a single fluid node. This simplifies the model by not requiring the use of parallel fluid elements. The matrix is populated with the area of the bimorph at the structural node row and the fluid node column.

\subsection{Damping}

\subsubsection{Fluid Damping}

Equation 33 contains a complete description of the finite element model for a fluid filled cavity driven by a simple piston type actuator. The only damping included in this model is the actuator damping matrix $C_{s}$; however, we know that the fluid must exert some damping effect on the vibrations of the system. The method adopted here is to substitute a complex speed of sound in the fluid mass matrix (equation 20) as described in Kinsler, et al. (1982). The speed of sound is written as

$$
\frac{1}{c^{*}}=\frac{1}{c}-j \frac{\alpha}{\omega}
$$

where $c^{*}$ is the complex speed of sound, $c$ is the thermodynamic speed of sound, $\omega$ is the frequency of the vibrations, and $\alpha$ is the absorption coefficient. 
The absorption coefficient is the sum of multiple absorption mechanisms. The predominant mechanisms are the absorption due to the bulk fluid, and absorption at the wall due to viscous resistance and thermal conduction. The bulk absorption is very much less than these two sources for frequencies less than approximately one $\mathrm{MHz}$ in air (Kinsler, 1982). Driving frequencies will be much less than one MHz in order to reduce the power requirements of the device, as the power consumed by a piezoelectric actuator is proportional to the driving frequency. The viscous and thermal effects on fluid motion in a pipe of radius $r$ result in an absorption coefficient of (Kinsler, 1982)

$$
\alpha=\frac{1}{r c} \sqrt{\frac{\eta_{e} \omega}{2 \rho_{f}}}
$$

where

$$
\eta_{\mathrm{e}}=\eta\left[1+(\gamma-1) \sqrt{\frac{\kappa}{C_{p} \eta}}\right]^{2}
$$

where $\eta$ and $\eta_{e}$ are the true and effective coefficients of shear viscosity, $\gamma$ is the ratio of specific heats, $\kappa$ is the thermal conductivity of the fluid, and $C_{p}$ is the specific heat at constant pressure. The absorption coefficient has been studied extensively resulting in models that consider the effects of humidity, temperature, pressure, and other complicating assumptions; see for example Rodarte, et al. (2000), Zuckerwar and Meredith (1985), Page and Mee (1984), and Tijdeman (1975). The additional complications were not deemed necessary, as the damping in the cavity will be adjusted at a later date once experimental data is available. The factor $1 / c^{2}$ in the finite element equations can be expanded now as

$$
\frac{1}{c^{2}} \Rightarrow \frac{1}{\left(c^{*}\right)^{2}}=\left(\frac{1}{c}-j \frac{\alpha}{\omega}\right)^{2}
$$


or

$$
\frac{1}{\left(c^{*}\right)^{2}}=\left(\frac{1}{c}\right)^{2}\left(1-j 2 \sqrt{\frac{\eta_{e}}{2 \rho_{f} \omega}}\left(\frac{1}{r}\right)-\left(\frac{\eta_{e}}{2 \rho_{f} \omega}\right)\left(\frac{1}{r^{2}}\right)\right)
$$

Substituting this result in equation 20 yields the following mass matrix for a fluid element

$$
\left[M_{f}\right]=\left(\frac{1}{c}\right)^{2} \int_{0}^{L}\left(1-j 2 \sqrt{\frac{\eta_{e}}{2 \rho_{f} \omega}}\left(\frac{1}{r}\right)-\left(\frac{\eta_{e}}{2 \rho_{f} \omega}\right)\left(\frac{1}{r^{2}}\right)\right) \rho(x)\{h\}^{T}\{h\} d x
$$

By examining the matrix described in equation 114 , we see that $\left[M_{f}\right]$ can be broken into three distinct matrices. Each matrix has geometric and frequency dependence. The geometric dependence can be evaluated during the creation of the finite element equations, but the frequency dependence must be calculated during the determination of the frequency response of the system. The following equations show this notation

$$
\left[M_{f}\right]=\frac{1}{c^{2}}\left[M_{f 1}-j 2 \sqrt{\frac{\eta_{e}}{2 \rho_{f} \omega}} M_{f 2}-\left(\frac{\eta_{e}}{2 \rho_{f} \omega}\right) M_{f 3}\right]
$$

where

$$
\begin{gathered}
M_{f 1}=\int_{0}^{L} \rho A(x)\{h\}^{T}\{h\} d x, \\
M_{f 2}=\int_{0}^{L}\left(\frac{1}{r}\right) \rho A(x)\{h\}^{T}\{h\} d x, \\
M_{f 3}=\int_{0}^{L}\left(\frac{1}{r^{2}}\right) \rho A(x)\{h\}^{T}\{h\} d x
\end{gathered}
$$$$
\text { and }
$$

With this formulation, the $M_{f 1}, M_{f 2}$, and $M_{f 3}$ matrices can be calculated for each element and formed into global matrices for the full system. During calculation of the frequency response, the full $\left[M_{f}\right]$ matrix is calculated depending on the frequency of interest. 
Alternatively, an acoustic boundary absorption coefficient can be assigned to the cavity surfaces. This is the approach used in the ANSYS program. Morse and Ingard (1968) give the following expression for the real part of the boundary absorption coefficient at the walls in a duct

$$
\beta=\frac{1}{2}\left(\frac{\omega}{c}\right)\left[d_{v}+(\gamma-1) d_{h}\right],
$$

where

$$
d_{v}=\sqrt{\frac{2 \eta}{\rho \omega}},
$$

and

$$
d_{h}=\sqrt{\frac{2 \kappa}{\rho \omega C_{p}}}
$$

The terms $d_{v}$ and $d_{h}$ are the thicknesses of the viscous and thermal boundary layers respectively, other symbols are as defined above. This equation is valid for ducts with radii greater than about three times the boundary layer thicknesses. By expanding these equations and comparing to the previous definition for absorption coefficient, we see that

$$
\alpha=\frac{1}{r} \beta
$$

In implementing this damping in ANSYS, an average value over the frequency range of interest is calculated and inserted in the ANSYS model. As each harmonic frequency is identified, the appropriate value for that frequency is used in equation 119. The resulting damping value is inserted back into ANSYS. This procedure is repeated until the natural frequency for all modes of interest is determined. 


\subsubsection{Damping in the piezoelectric actuator}

Data sheets for the PZT material specify damping in terms of the mechanical $Q$. This mechanical $Q$ can be considered either hysteretic or viscous damping as follows (Nashif, 1985).

$$
\begin{aligned}
& Q_{M}=\frac{1}{2 \zeta} \quad \text { (viscous) } \\
& Q_{M}=\frac{1}{\eta_{L}} \quad \text { (hysteretic) }
\end{aligned}
$$

For viscous damping, the term $\zeta$ is the damping ratio. The hysteretic damping term is the ratio of real to imaginary parts of the stiffness and is known as the loss factor.

$$
K^{*}=K\left(1+i \eta_{L}\right)
$$

where $K^{*}$ is the complex stiffness and $K$ is the real part. The hysteretic damping is a more physical approach for the piezoelectric material and is easily incorporated in the Matlab routine by multiplying the " $c$ " matrix portion of the stiffness matrix. ANSYS does not allow hysteretic damping; therefore, the damping ratio must be used in ANSYS. The addition of viscous damping to an undamped system decreases both the amplitude and resonant frequency of the system. Hysteretic damping will only affect the amplitude of the vibration at resonance. This will result in the frequency calculated by ANSYS to be somewhat less than that determined by the Matlab solution. The error should be minimal as the damping is so small that the differences will generally be negligible. 


\subsection{Implementation and Optimization in Matlab}

The preceding equations were incorporated in a MATLAB program. Matlab was chosen as it is easy to program, high level language, and it has toolboxes available that allow the use of symbolic notation and optimization. This section describes the procedure used to solve the equations of the preceding sections.

The acoustic cavity with bimorph actuator is shown schematically in Figure 12. The fluid-structure interaction is between the odd numbered piezoelectric nodes and the first fluid node. The odd numbered nodes are the translation and the even numbered nodes are the rotation degrees of freedom for the element.

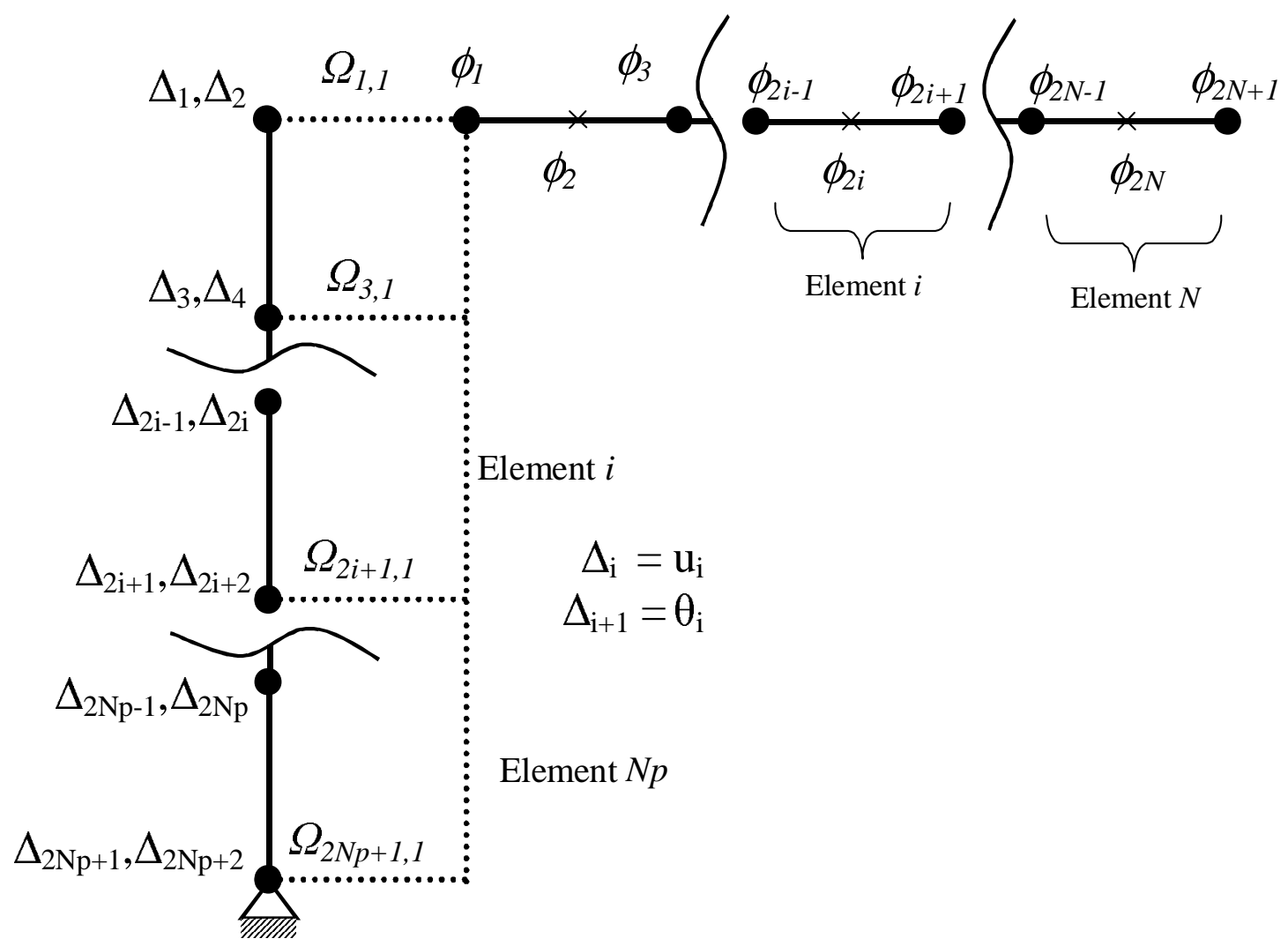

Figure 12 - Finite Element Model of Acoustic Cavity with Bimorph Actuator 
The interaction area at each node is calculated by using half an element length on either side of the node to determine inner and outer radii. The exceptions are the end nodes which only use the side where an element exists to determine the interaction area. For the stacked actuator, the piezoelectric and fluid elements only interact at one node and the area is the full diameter of the stack.

With the element scheme from Figure 12 and the equations of the preceding sections, the finite element matrices can be computed. The complete equations of motion describing the piezoelectric actuator interacting with the acoustic fluid are shown below in expanded form.

$$
\left[\begin{array}{ccc}
M_{u u} & 0 & 0 \\
0 & 0 & 0 \\
0 & 0 & -M_{f}
\end{array}\right]\left\{\begin{array}{l}
\ddot{\Delta}_{u} \\
\ddot{\Delta}_{d} \\
\ddot{\Phi}
\end{array}\right\}+\left[\begin{array}{ccc}
C_{u u} & 0 & \Omega \\
0 & 0 & 0 \\
\Omega^{T} & 0 & 0
\end{array}\right]\left\{\begin{array}{c}
\dot{\Delta}_{u} \\
\dot{\Delta}_{d} \\
\dot{\Phi}
\end{array}\right\}+\left[\begin{array}{ccc}
K_{u u} & -K_{u d} & 0 \\
-K_{u d}^{T} & K_{d d} & 0 \\
0 & 0 & -K_{f}
\end{array}\right]\left\{\begin{array}{c}
\Delta_{u} \\
\Delta_{d} \\
\Phi
\end{array}\right\}=\left\{\begin{array}{c}
0 \\
Q_{n c} \\
0
\end{array}\right\}
$$

The fluid damping embedded in the $M_{f}$ matrix is a function of the driving frequency; therefore, an iterative solution scheme must be used. A brute force method of solution could be employed where the solution over a frequency range is obtained and then a peak search done to find the resonant frequencies. During optimization, this search method would result in very long run times for each iteration of the cavity. A better approach is to estimate the natural frequencies of the system, then perform a search about the estimate to locate the true natural frequency. The Matlab control toolbox provides the "damp" command to determine the natural frequencies of a damped first order linear differential equation system. The second order equation can be recast into a first order system by the substitution of a new coordinate. 


$$
\{\dot{X}\}=\left[\begin{array}{cc}
0 & I \\
-[M]^{-1}[K] & -[M]^{-1}[C]
\end{array}\right]\{X\},
$$

where

$$
\{X\}=\left\{\begin{array}{l}
\Delta \\
\dot{\Delta}
\end{array}\right\}
$$

and the matrices have been collapsed for clarity. The difficulty with employing this method is that the electrical degrees of freedom have no entry in the mass matrix, resulting in an indeterminate inverse. The first step in solving this problem is to reduce the order of the piezoelectric matrices to condense out the electrical degrees of freedom. Expand the matrix equations for the piezoelectric actuator

$$
M_{u u} \ddot{\Delta}_{u}+C_{u u} \dot{\Delta}_{u}+K_{u u} \Delta_{u}-K_{u d} \Delta_{d}=0
$$

and

$$
-K_{u d}^{T} \Delta_{u}+K_{d d} \Delta_{d}=Q_{n c}
$$

and solve the second set of equations for the electrical displacement

$$
\Delta_{d}=K_{d d}^{-1} Q_{n c}+K_{d d}^{-1} K_{u d}^{T} \Delta_{u}
$$

Substitute this equation back into the first set of matrix equations

$$
\begin{gathered}
M_{u u} \ddot{\Delta}_{u}+C_{u u} \dot{\Delta}_{u}+K_{u u} \Delta_{u}-K_{u d}\left(K_{d d}^{-1} Q_{n c}+K_{d d}^{-1} K_{u d}^{T} \Delta_{u}\right)=0, \\
\text { or } \quad M_{u u} \ddot{\Delta}_{u}+\left(K_{u u}-K_{u d} K_{d d}^{-1} K_{u d}^{T}\right) \Delta_{u}=K_{u d} K_{d d}^{-1} Q_{n c}
\end{gathered}
$$

The reduced order model for the piezoelectric actuator is

$$
M R \ddot{\Delta}_{u}+C R \dot{\Delta}_{u}+K R \Delta_{u}=F R
$$

where

$$
\begin{gathered}
M R=M_{u u}, \\
C R=C_{u u}, \\
K R=K_{u u}-K_{u d} K_{d d}^{-1} K_{u d}^{T},
\end{gathered}
$$

and

$$
F R=K_{u d} K_{d d}^{-1} Q_{n c}
$$

The resulting reduced order system model is now 


$$
\left[\begin{array}{cc}
M R & 0 \\
0 & -M_{f}
\end{array}\right]\left\{\begin{array}{c}
\ddot{\Delta}_{u} \\
\ddot{\Phi}
\end{array}\right\}+\left[\begin{array}{cc}
C R & \Omega \\
\Omega^{T} & 0
\end{array}\right]\left\{\begin{array}{c}
\dot{\Delta}_{u} \\
\dot{\Phi}
\end{array}\right\}+\left[\begin{array}{cc}
K R & 0 \\
0 & -K_{f}
\end{array}\right]\left\{\begin{array}{c}
\Delta_{u} \\
\Phi
\end{array}\right\}=\left\{\begin{array}{c}
F R \\
0
\end{array}\right\}
$$

Since the actuator damping is embedded in the $K R$ matrix, $C R$ is the appropriate size zero matrix. The above matrices can be used in equation 127 without any difficulties.

The fluid mass matrix is calculated at a trial frequency of $1000 \mathrm{~Hz}$ then the estimates of the natural frequency are calculated from the reduced order system. These estimates are used as the start point for a simple search to determine the peak pressure response at the end of the fluid cavity. This position was chosen because it is the preferred location for the valves that allow the acoustical compressor to deliver the high-pressure fluid.

The entire resonator drive scheme was also implemented in the Matlab code to allow comparison with published experimental data. Lawrenson, et al. (1998) report results for standing waves in closed cavities generated by oscillating the entire resonator using an electrodynamic shaker. The resonators were filled with refrigerant R-134A (1,1,1,2tetrafluoroethane). The results reported in the paper do not include the velocity used to drive the resonators, only the power delivered to them. The average power delivered to the fluid per cycle is (Ilinskii, et al., 2001)

$$
\text { Power }=\int_{V} \rho a v d V
$$

where $a$ is the imposed acceleration on the resonator and $v$ is the velocity of the fluid. When entire resonator drive is chosen, the Matlab routine prints the power delivered to the fluid by the imposed acceleration. Using this information, the input acceleration may be varied until the proper input power is reached. 
A large number of fluid elements will be required to adequately capture the geometry and response of the fluid cavity. This large number of shape variables will present a difficult problem for the optimizer and result in very long run times if convergence can be obtained at all. For this reason, the shape of the cavity is generated by a cubic polynomial fit between a few control points. The polynomial is used to discretize the cavity into an arbitrary number of fluid elements while using only a few controlling variables. This method reduces the number of variables in the optimization.

The code does not include modeling of fluid flow effects; therefore, these were accounted for by constraining the shape of the cavity. Expansion sections in the cavity will result in an adverse pressure gradient that can cause separation of the flow and result in turbulence in that area. The maximum divergence angle was restricted to 10 degrees to ensure the fluid flow would remain in the laminar range (Mehta and Bradshaw, 1979). The objective of the optimization was to generate maximum pressure at the end of the cavity for minimum power input. The figure of merit used to judge a cavity was the end pressure minus $10 \%$ of the electrical power required to drive the cavity. Furthermore, if the velocity of the fluid exceeded 0.98 of the speed of sound, the figure of merit was set to zero. Although the true fluid behavior is strongly nonlinear at high pressures and velocities, the use of these penalty functions in the optimization prevents the routine from finding solutions that would result in shock wave formation. 


\subsection{Nonlinear Effects}

The finite element equations developed in this thesis are linear in nature. As noted in section 2.1, the fluid must be inviscid, isotropic, and undergoing small displacements. The inviscid requirement is minimized due to the inclusion of damping in the form of a complex velocity with absorption coefficient. Most fluids of interest for this application behave isotropicaly, so this is a good assumption. The difficulty with the linear equations is that we are using them to design a cavity that obviously causes large displacements and velocities to create the high pressure. The development and solution of the non-linear finite element equations describing the fluid motion is beyond the scope of this thesis. However, a discussion of them is in order.

One formulation of the nonlinear fluid finite element equation is shown in equation 134 (Hoffelner, et al.2001)

$$
\left[M_{f}\right]\{\ddot{\Phi}\}+\left[C_{f}\right]\{\dot{\Phi}\}+\left[K_{f}\right]\{\Phi\}=\left[N_{1}(\dot{\Phi})\right]\{\ddot{\Phi}\}+\left[N_{2}(\Phi)\right]\{\dot{\Phi}\}
$$

where $M, C$, and $K$ are the linear mass, damping, and stiffness matrices. The two nonlinear matrices on the right hand side are

$$
\begin{aligned}
& \left.\left[N_{1}(\dot{\Phi})\right]=\int_{0}^{L} \rho A(x) \frac{B}{A} \frac{1}{c^{4}}\{h\}\{\dot{\Phi}\} h\right\}^{T}\{h\} d x \\
& \left.\left.\left[N_{2}(\Phi)\right]=\int_{0}^{L} \rho A(x) \frac{2}{c^{2}}\left\{h_{x}\right\} \Phi\right\} h\right\}^{T}\left\{h_{x}\right\} d x
\end{aligned}
$$

where $B / A$ is known in the literature on nonlinear acoustics as the nonlinearity parameter. For an ideal gas, 


$$
\frac{B}{A}=\gamma-1
$$

The $N_{l}$ matrix will be small if either the nonlinearity parameter is small or the pressure is low ( $p=-\rho\{h\}\{\dot{\Phi}\})$. The $N_{2}$ matrix will only be small for low velocities ( $\left.\left.v=\left\{h_{x}\right\} \Phi\right\}\right)$. Using a nonlinear solution approach and these equations would have resulted in excessively long run times during the optimization. The linear solution gives a good approximation to the solution and can be used to evaluate the relative performance of different shapes for the cavity. As will be seen in the results section, the linear code provides useful results. 


\section{Results}

The simple fluid - structure interaction described in section 2.1 was first coded and verified. Verification was done by comparing the pressures calculated against an ANSYS model. Then the stacked actuator of section 2.2.2 was added and verified.

Finally, the bimorph model was verified on its own and then added to the fluid-structure interaction. Once the routine was verified, the MATLAB optimization functions were used to generate an optimum actuator/cavity configuration.

The material properties used are shown in Table 2 (Morgan Electro Ceramics, and Kays and Crawford 1993).

Table 2 - Material Properties

\begin{tabular}{|l|c|l|l|l|l|}
\hline \multicolumn{2}{|l|}{ Piezoelectric Material (PZT-5A) } & \multicolumn{3}{l|}{ Fluid Material (Air) } \\
\hline Property & Value & Units & Property & Value & Units \\
\hline$e_{31}^{E}$ & -5.4 & $\frac{N}{\mathrm{volt} \cdot \mathrm{m}}$ & $\rho$ & 1.225 & $\frac{\mathrm{kg}}{\mathrm{m}^{3}}$ \\
\hline$e_{33}^{E}$ & 15.8 & $\frac{\mathrm{N}}{\mathrm{volt} \cdot \mathrm{m}}$ & $\mathrm{c}$ & 340.3 & $\frac{\mathrm{m}}{\mathrm{s}}$ \\
\hline$c_{11}^{E}$ & 121.0 & $\mathrm{GPa}$ & $\gamma$ & 1.4 & \\
\hline$c_{12}^{E}$ & 75.4 & $\mathrm{GPa}$ & $C_{p}$ & 1004 & $\frac{\mathrm{J}}{\mathrm{kg} \cdot \mathrm{K}}$ \\
\hline$c_{13}^{E}$ & 75.2 & $\mathrm{GPa}$ & $\kappa$ & 25.36 & $\frac{\mathrm{mW}}{\mathrm{m} \cdot \mathrm{K}}$ \\
\hline$c_{33}^{E}$ & 111.0 & $G P a$ & $\eta$ & 17.89 & $\mu P a \cdot s$ \\
\hline$\rho$ & 7750 & $\frac{\mathrm{kg}}{\mathrm{m}^{3}}$ & & & \\
\hline $\mathrm{Q}_{\mathrm{M}}$ & 75 & & & & \\
\hline$\varepsilon_{33}^{S} / \varepsilon_{0}$ & 830 & & & & \\
\hline
\end{tabular}




\subsection{Verification of Acoustic Fluid FEM and Damping}

As a first step prior to optimization, the MATLAB program was verified against a simple tapered cavity shown in Figure 13. The purpose of this verification exercise was to determine that the equations for the acoustic fluid interacting with a moving structure and incorporating fluid damping would produce accurate results when compared against the commercial FEM code ANSYS.

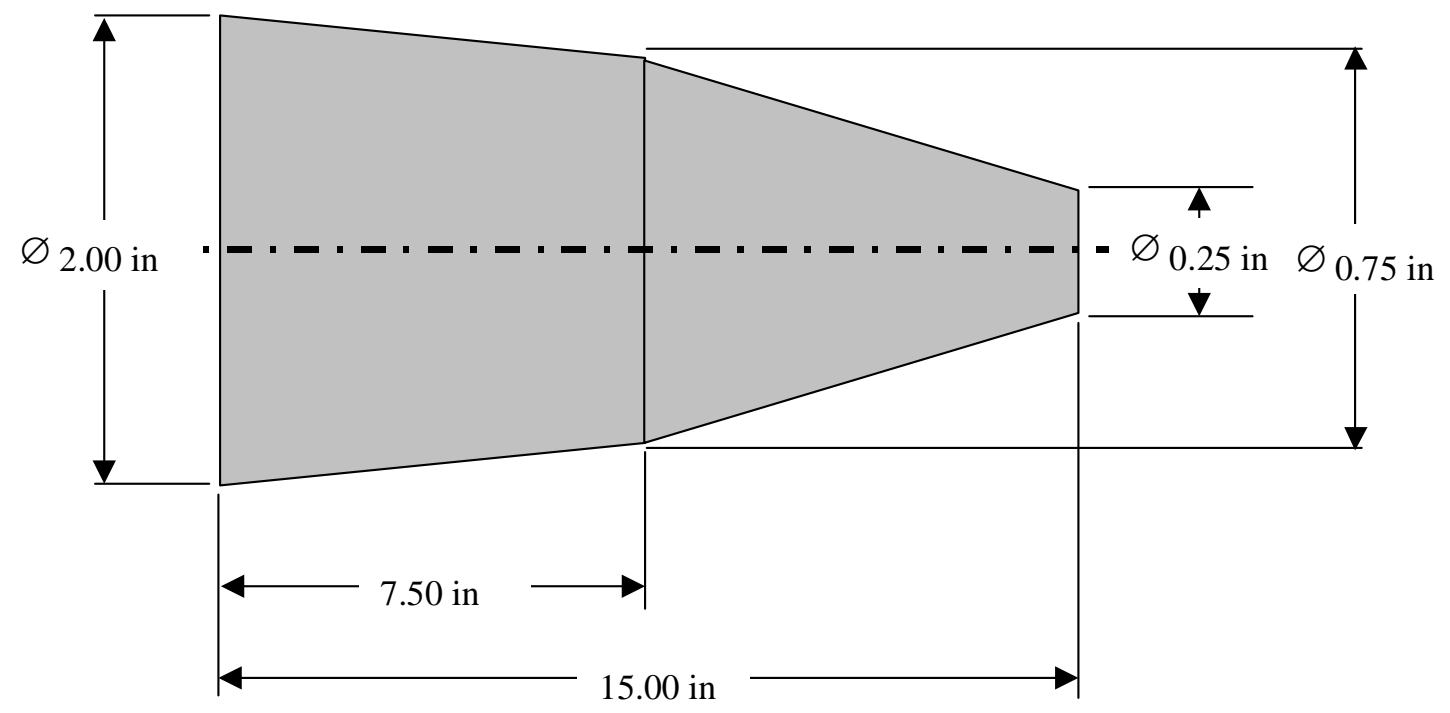

Figure 13 - Verification Model Cavity

The ANSYS model is a two-dimensional axisymmetric model of the fluid cavity. The stacked piezoelectric actuator was not incorporated in the ANSYS model; an imposed deflection matching that of the MATLAB model was placed on the boundary representing the actuator interface. The MATLAB model used 48 fluid elements, while the ANSYS model was 1870 fluid elements. A comparison of the results from the two 
approaches is shown below in Figure 14 and Table 3. The "End Pressure" in Table 3 refers to one half the peak-to-peak pressure over an acoustic cycle.

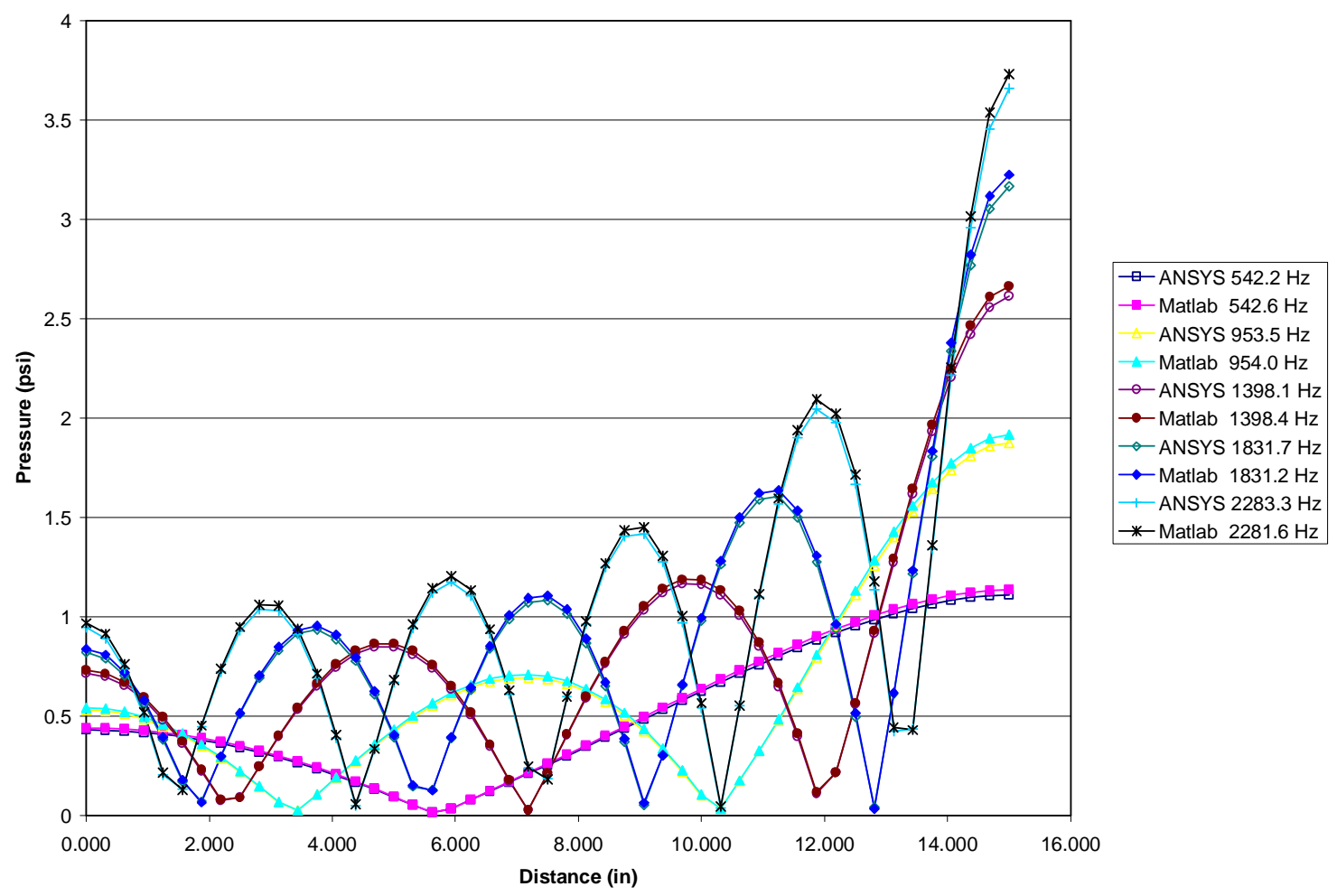

Figure 14 - Comparison of Matlab and ANSYS Solutions of Verification Model Cavity

Table 3 - Frequency and End Pressure Comparison of Verification Model Cavity

\begin{tabular}{|c|r|r|r|r|r|r|}
\hline \multirow{2}{*}{$\begin{array}{c}\text { Mode } \\
\text { Number }\end{array}$} & \multicolumn{3}{|c|}{ Frequency (Hz) } & \multicolumn{3}{c|}{ End Pressure (psi) } \\
\cline { 2 - 7 } & Matlab & ANSYS & Difference & Matlab & ANSYS & Difference \\
\hline 1 & 542.57 & 542.23 & $0.1 \%$ & 1.13 & 1.11 & $2.2 \%$ \\
\hline 2 & 954.05 & 953.53 & $0.1 \%$ & 1.91 & 1.88 & $2.1 \%$ \\
\hline 3 & 1398.37 & 1398.13 & $0.0 \%$ & 2.66 & 2.61 & $1.9 \%$ \\
\hline 4 & 1831.24 & 1831.66 & $0.0 \%$ & 3.23 & 3.17 & $1.9 \%$ \\
\hline 5 & 2281.59 & 2283.30 & $0.1 \%$ & 3.73 & 3.66 & $2.0 \%$ \\
\hline
\end{tabular}


The mode shapes along the centerline agree quite well and the frequency agreement is also excellent. There is a small difference in calculated end pressure, likely due to the one-dimensional model versus a two-dimensional model. Increasing the number of elements in the Matlab model up to 200 elements made no appreciable difference to the results.

\subsection{Verification of Bimorph FEM}

A 480 element 2-D axisymmetric ANSYS model was created to verify the frequencies and displacements calculated by a 48 element 1-D Matlab model. The actuator modeled consisted of a PZT-5H bimorph of radius 1.25 inch, layer thickness $0.19 \mathrm{~mm}$, actuation voltage 90 volts across each layer. Figure 15 below shows a comparison of the mode shapes and displacements. Table 4 and Table 5 compare the frequency, displacement, and drive power required assuming that the ANSYS result is more correct. 


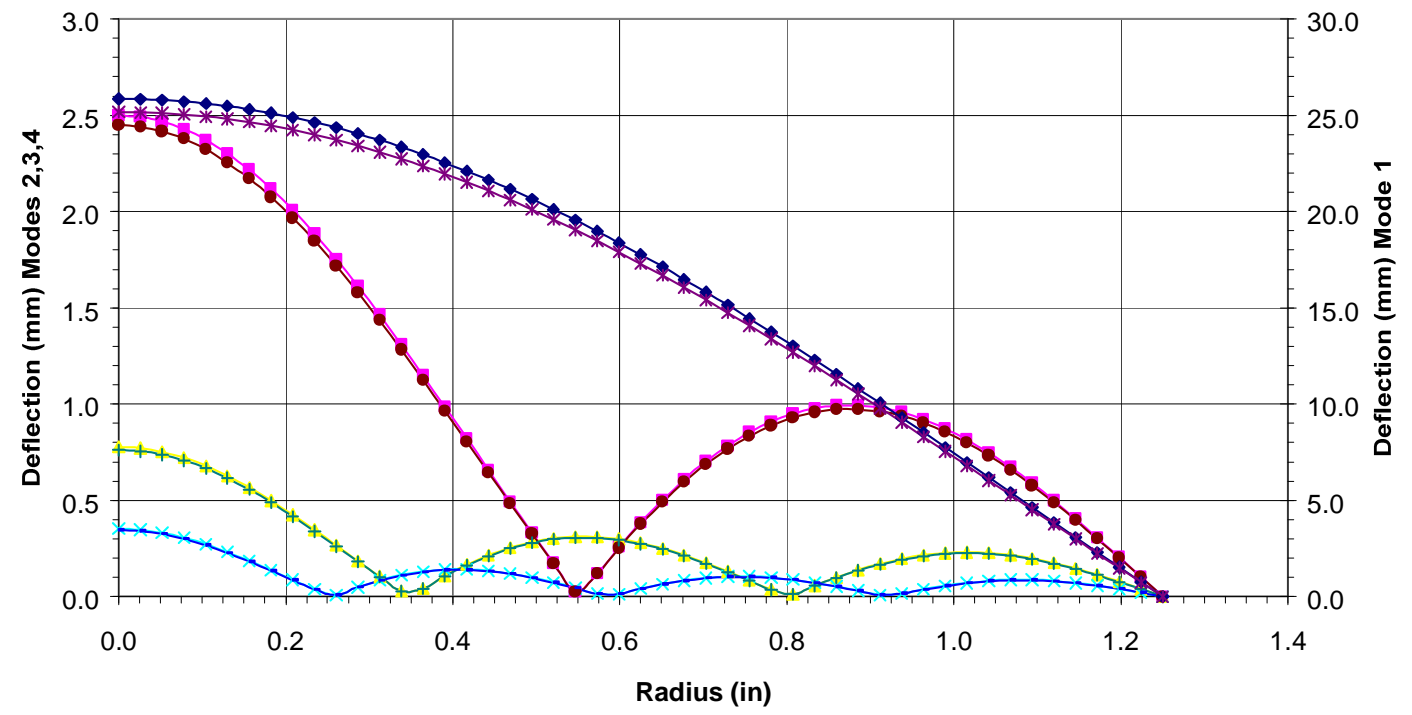

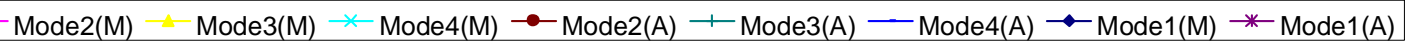

Figure 15 - Bimorph Results Comparison, Matlab (M) vs. ANSYS (A)

Table 4 - Bimorph Model Frequency and Displacement Comparison

\begin{tabular}{|c|r|r|r|r|r|r|}
\hline \multirow{2}{*}{$\begin{array}{c}\text { Mode } \\
\text { Number }\end{array}$} & \multicolumn{3}{|c|}{ Frequency (Hz) } & \multicolumn{3}{c|}{ Peak displacement (mm) } \\
\cline { 2 - 7 } & MATLAB & ANSYS & Difference & MATLAB & ANSYS & Difference \\
\hline Static & & & & 0.281 & 0.285 & $1.4 \%$ \\
\hline 1 & 277.4 & 275.5 & $0.7 \%$ & 25.856 & 25.177 & $2.7 \%$ \\
\hline 2 & 1627.0 & 1618.2 & $0.5 \%$ & 2.500 & 2.450 & $2.0 \%$ \\
\hline 3 & 4049.5 & 4026.8 & $0.6 \%$ & 0.777 & 0.762 & $1.9 \%$ \\
\hline 4 & 7547.7 & 7502.8 & $0.6 \%$ & 0.353 & 0.346 & $1.8 \%$ \\
\hline
\end{tabular}

Table 5 - Bimorph Model Drive Power Comparison

\begin{tabular}{|c|r|r|r|}
\hline \multirow{2}{*}{$\begin{array}{c}\text { Mode } \\
\text { Number }\end{array}$} & \multicolumn{3}{|c|}{ Drive Power (watts) } \\
\cline { 2 - 4 } & MATLAB & ANSYS & Difference \\
\hline 1 & 109.9 & 106.5 & $3.2 \%$ \\
\hline 2 & 91.5 & 89.4 & $2.3 \%$ \\
\hline 3 & 107.3 & 105.6 & $1.6 \%$ \\
\hline 4 & 149.6 & 148.1 & $1.0 \%$ \\
\hline
\end{tabular}


The agreement is excellent, considering that the MATLAB model has $1 / 10$ of the number of elements and fewer degrees of freedom than the ANSYS model. Notice that for a relatively low power input, the bimorph delivers an impressive amount of deflection compared to a stacked type actuator. At these large deflections, the model assumptions are violated and the solution accuracy is decreased. The bimorph would likely break without some type of reinforcing layer.

3.3 Verification of Bimorph FEM with fluid and damping in a sample cavity

Finally, the bimorph model was added to the fluid model and verified using the cavity in Figure 13. The results of the ANSYS model of the tapered cavity versus the Matlab solution are shown in Table 6 and Table 7.

Table 6 -Tapered PAC Model Frequency and End Pressure Comparison

\begin{tabular}{|c|r|r|r|r|r|r|}
\hline \multirow{2}{*}{$\begin{array}{c}\text { Mode } \\
\text { Number }\end{array}$} & \multicolumn{3}{|c|}{ Frequency } & \multicolumn{3}{c|}{ End Pressure (psi) } \\
\cline { 2 - 7 } & MATLAB & ANSYS & Difference & MATLAB & ANSYS & Difference \\
\hline 1 & 426.71 & 424.16 & $0.6 \%$ & 3.38 & 3.24 & $4.3 \%$ \\
\hline 2 & 548.77 & 548.41 & $0.1 \%$ & 3.69 & 3.62 & $1.9 \%$ \\
\hline 3 & 944.15 & 948.71 & $0.5 \%$ & 0.918 & 0.924 & $0.6 \%$ \\
\hline 4 & 1388.51 & 1388.70 & $0.0 \%$ & 0.349 & 0.358 & $2.7 \%$ \\
\hline
\end{tabular}


Table 7 -Tapered PAC Model Displacement and Drive Power Comparison

\begin{tabular}{|c|r|r|r|r|r|r|}
\hline \multirow{2}{*}{$\begin{array}{c}\text { Mode } \\
\text { Number }\end{array}$} & \multicolumn{2}{|c|}{ Center point displacement (um) } & \multicolumn{2}{c|}{ Drive Power (watts) } \\
\cline { 2 - 7 } & MATLAB & ANSYS & Difference & MATLAB & ANSYS & Difference \\
\hline 1 & 15198.90 & 14876.00 & $2.2 \%$ & 99.43 & 96.90 & $2.6 \%$ \\
\hline 2 & 1220.80 & 1175.85 & $3.8 \%$ & 10.56 & 10.22 & $3.4 \%$ \\
\hline 3 & 78.67 & 78.39 & $0.4 \%$ & 10.04 & 10.12 & $0.8 \%$ \\
\hline 4 & 44.36 & 44.70 & $0.8 \%$ & 15.57 & 15.60 & $0.2 \%$ \\
\hline
\end{tabular}

The frequency agreement of the combined model is still excellent. The results agree to within $4 \%$ on amplitude and less than $1 \%$ on frequency even though different damping methods are used and the MATLAB model has many fewer elements. The end pressure and drive power calculated are also in good agreement.

\subsection{Comparison with Published Measurement Data}

Lawrenson, et al. (1998) report the pressure measured at the end of several different geometry resonators filled with refrigerant R-134A and employing Entire Resonator Drive (ERD). They analyzed the pressure signal and give the pressure recorded for the first 10 harmonics of the fundamental frequency used to drive the resonator. They considered several geometries, a cylinder, a cone, and a horn-cone shape. They only report both pressure and power for the horn-cone; therefore, this geometry was used to verify the Matlab model against a real world problem. The acceleration in the model was varied until the output power matched that reported in the paper. 
The thermodynamic properties of the refrigerant used in the MATLAB calculations are shown in Table 8. The paper lists the density of the R-134A used in the experiment as $13.8 \mathrm{~kg} / \mathrm{m}^{3}$ and gives the ambient pressure within the cavity for the time during which measurements were taken. Since the cavities were sealed, the ambient density does not change from one experiment to the next. Given the density and pressure, the other thermodynamic properties were calculated. These properties are obtained from the software program EES that implements the Martin-Hou equation of state.

Table 8 - Material Properties for R-134A used in Experiment

\begin{tabular}{|c|c|c|c|}
\hline Property & $\mathrm{P}=306 \mathrm{kPa}$ & $\mathrm{P}=327 \mathrm{kPa}$ & Units \\
\hline$\rho$ & 13.8 & 13.8 & $\frac{\mathrm{kg}}{\mathrm{m}^{3}}$ \\
\hline$c$ & 154 & 159.2 & $\frac{\mathrm{m}}{\mathrm{s}}$ \\
\hline$\gamma$ & 1.16 & 1.16 & \\
\hline$C_{p}$ & 908.4 & 922.2 & $\frac{\mathrm{J}}{\mathrm{kg} \cdot \mathrm{K}}$ \\
\hline$\kappa$ & 13.77 & 15.05 & $\frac{\mathrm{mW}}{\mathrm{m} \cdot \mathrm{K}}$ \\
\hline$\eta$ & 11.75 & 12.41 & $\mu \mathrm{Pa} \cdot \mathrm{s}$ \\
\hline
\end{tabular}



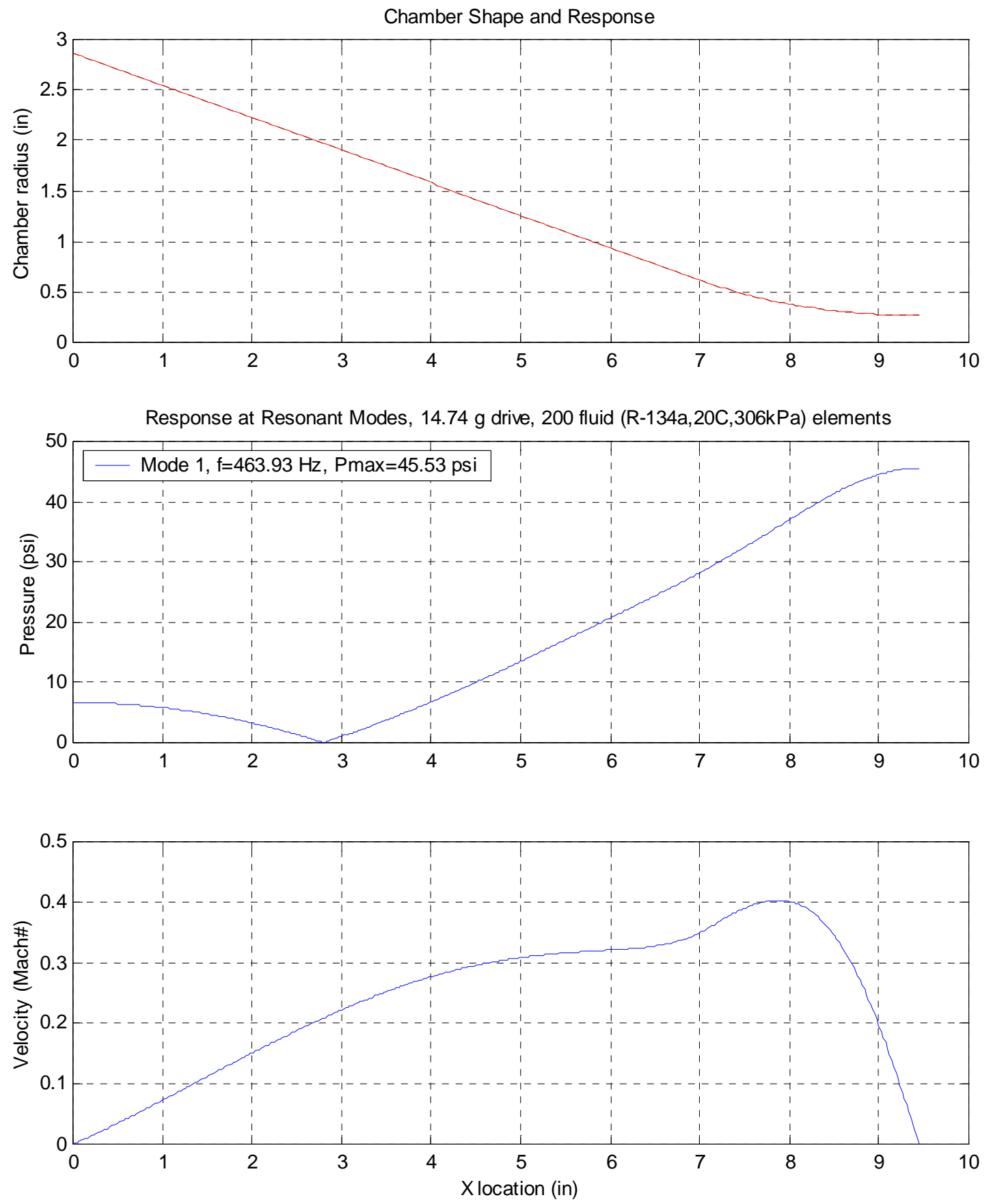

Figure 16 -MATLAB output for horn-cone geometry using ERD at 14.74g (41.7 Watts) 

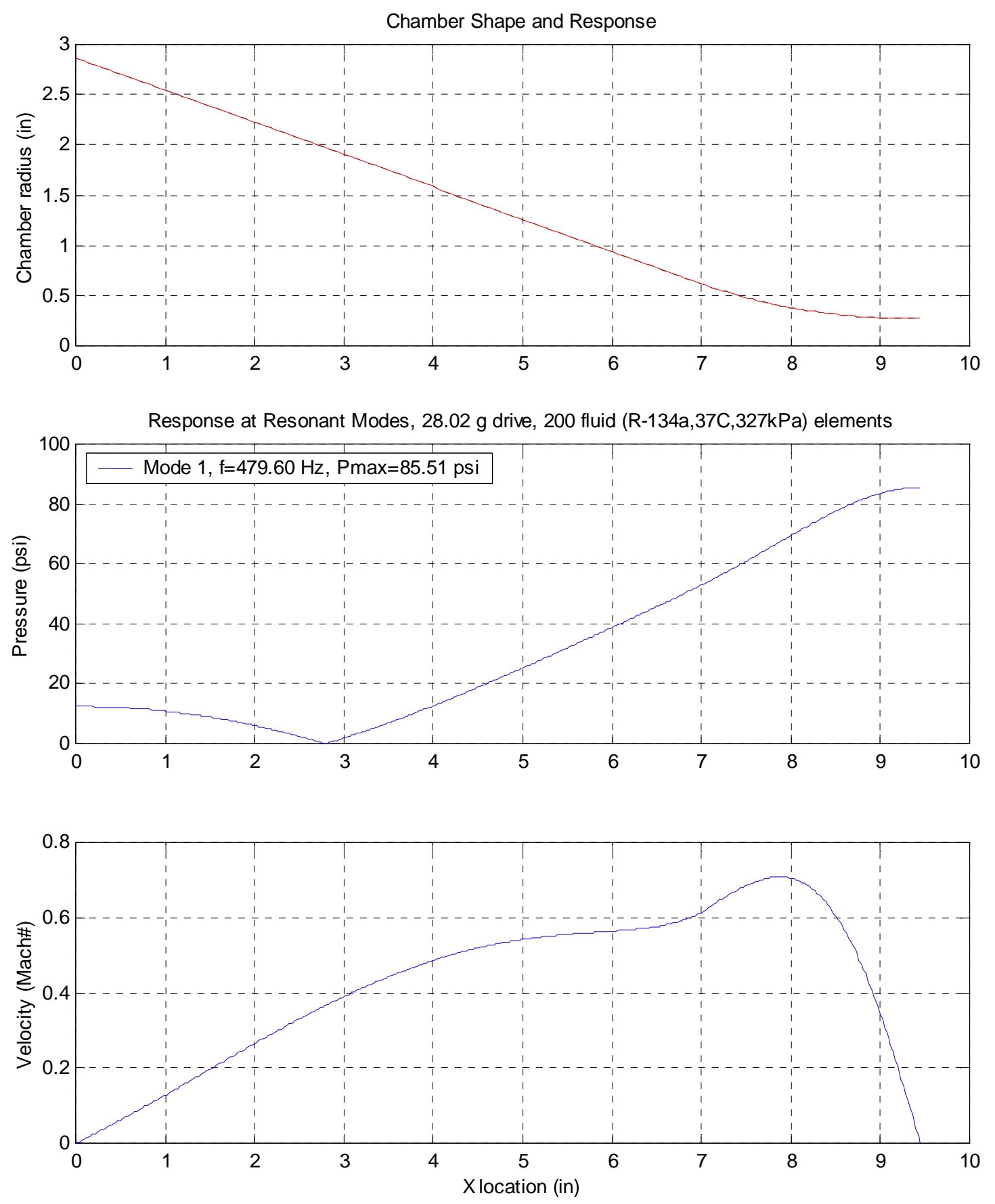

Figure 17 -MATLAB output for horn-cone geometry using ERD at 28.02g (144 Watts) 


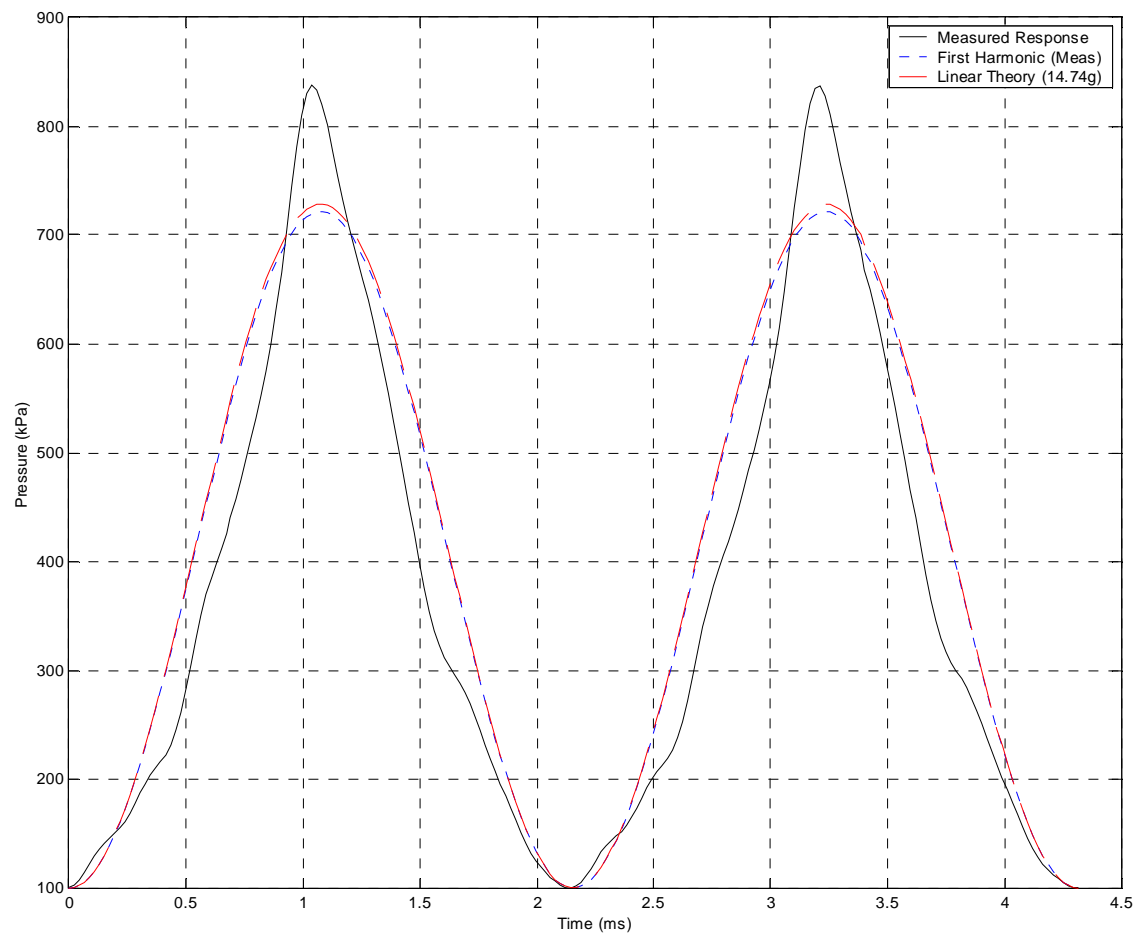

Figure 18 - Time History Comparison of MATLAB to Experiment (41.7 Watts)

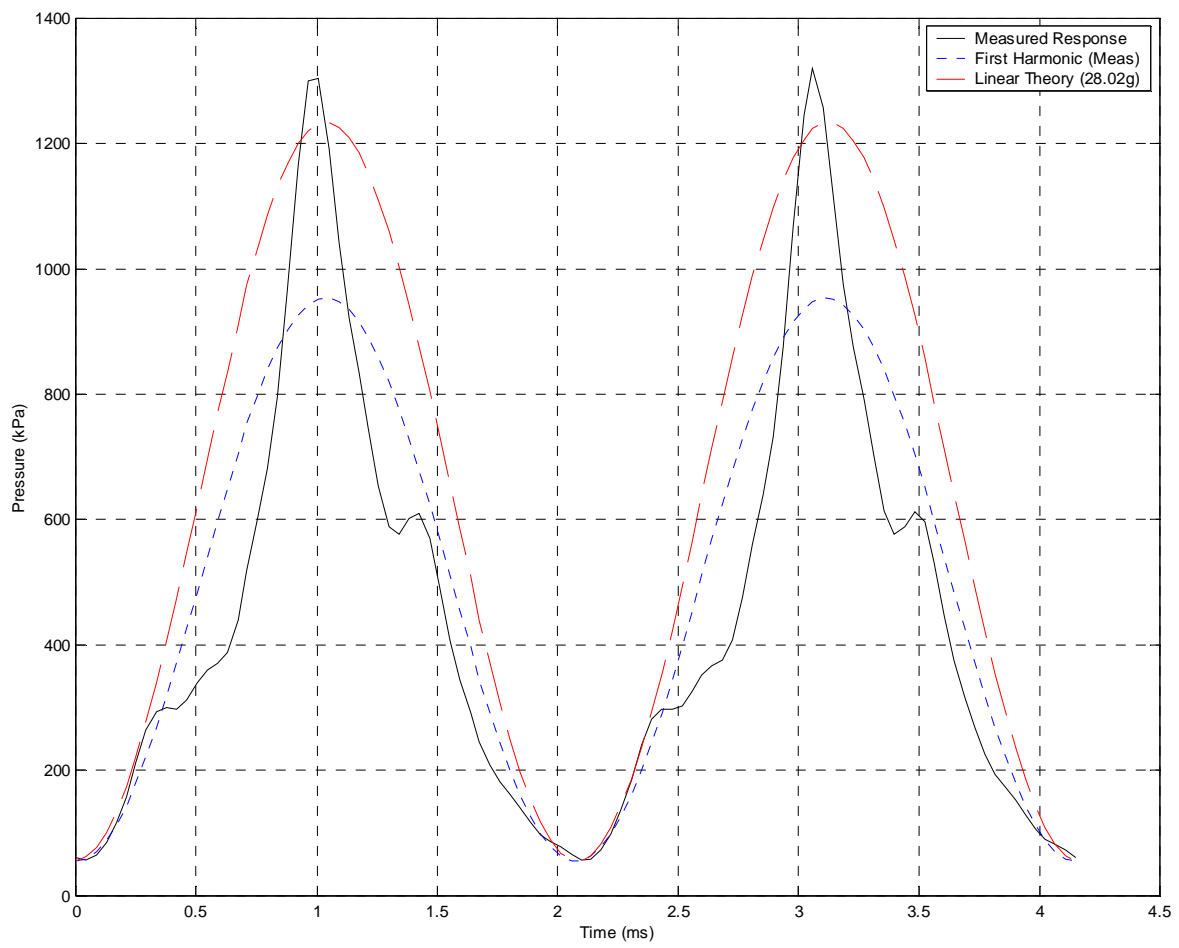

Figure 19 - Time History Comparison of MATLAB to Experiment (144 Watts) 
Table 9 - Comparison of Matlab Linear Code to Experiment

\begin{tabular}{|c|r|r|r|r|r|r|}
\hline Power & \multicolumn{3}{|c|}{ Frequency (Hz) } & \multicolumn{3}{c|}{ Peak to Peak Pressure (psi) } \\
(Watts) & Experiment & MATLAB & Difference & Experiment & MATLAB & Difference \\
\hline 41.7 & 463.6 & 463.9 & $0.1 \%$ & 106.8 & 91.1 & $14.7 \%$ \\
\hline 144 & 481.8 & 479.6 & $0.5 \%$ & 183.5 & 171.0 & $6.8 \%$ \\
\hline
\end{tabular}

Table 10 - Comparison of Matlab Linear Code to Experiment (First Harmonic)

\begin{tabular}{|c|r|r|r|}
\hline \multirow{2}{*}{$\begin{array}{c}\text { Power } \\
\text { (Watts) }\end{array}$} & \multicolumn{3}{|c|}{$\begin{array}{r}\text { Peak to Peak Pressure (psi) } \\
\text { First Harmonic Only }\end{array}$} \\
\hline 41.7 & Experiment & MATLAB & Difference \\
\hline 144 & 130.1 & 91.1 & $1.1 \%$ \\
\hline
\end{tabular}

Table 9 and Table 10 summarize the results compared to the experiment. Figure 16 and Figure 17 show the shape of the standing wave and fluid velocity along the resonator axis for different drive levels. Time history plots of the pressure signal at the end of the horn cone geometry in Figure 18 and Figure 19 compare the Matlab solution to the total measured response and the first harmonic measured response. The Matlab results have been adjusted in level to coincide with the lowest pressure measured in the experiment. The Matlab solutions assume all of the input energy is delivered to the first harmonic; however, the nonlinearities present in the system pump some of the energy into higher harmonics. This is particularly evident in Figure 19, where the linear solution is considerably higher in level than the actual first harmonic measured. 
The Matlab code was also compared to an ANSYS model. The two solutions are compared using the same accelerations and material properties as were used in the experiment comparisons above. Table 11 compares the results for $14.74 \mathrm{~g}$ acceleration and Table 12 for $28.02 \mathrm{~g}$. The percent difference at each mode for the frequency and end pressure is exactly the same between the two drive levels. This seems to point to a systematic modeling difference rather than some error in the code. As the Matlab frequency agreement is excellent with the experiment, it appears that the Matlab model accurately models the frequency response of the fluid.

Table 11 - Comparison of Matlab Linear Code to ANSYS for 14.74g Acceleration of Horn-Cone Resonator

\begin{tabular}{|c|r|r|r|r|r|r|}
\hline Mode & \multicolumn{3}{|c|}{ Frequency (Hz) } & \multicolumn{3}{c|}{ End Pressure (psi) } \\
Number & MATLAB & ANSYS & Difference & MATLAB & ANSYS & Difference \\
\hline 1 & 463.9 & 452.9 & $2.4 \%$ & 45.5 & 43.7 & $4.1 \%$ \\
\hline 2 & 725.9 & 713.3 & $1.7 \%$ & 15.9 & 15.2 & $4.2 \%$ \\
\hline 3 & 990.9 & 973.7 & $1.7 \%$ & 15.4 & 14.6 & $5.2 \%$ \\
\hline 4 & 1306.6 & 1274.8 & $2.4 \%$ & 11.4 & 9.7 & $14.6 \%$ \\
\hline
\end{tabular}

Table 12 - Comparison of Matlab Linear Code to ANSYS for 28.02g Acceleration of Horn-Cone Resonator

\begin{tabular}{|c|r|r|r|r|r|r|}
\hline Mode & \multicolumn{3}{|c|}{ Frequency (Hz) } & \multicolumn{3}{c|}{ End Pressure (psi) } \\
Number & MATLAB & ANSYS & Difference & MATLAB & ANSYS & Difference \\
\hline 1 & 479.6 & 468.2 & $2.4 \%$ & 85.5 & 82.0 & $4.1 \%$ \\
\hline 2 & 750.4 & 737.4 & $1.7 \%$ & 29.9 & 28.6 & $4.2 \%$ \\
\hline 3 & 1024.4 & 1006.6 & $1.7 \%$ & 28.8 & 27.4 & $5.1 \%$ \\
\hline 4 & 1350.7 & 1317.8 & $2.4 \%$ & 21.4 & 18.2 & $14.6 \%$ \\
\hline
\end{tabular}


The frequency and pressure calculated by the linear code are in reasonable agreement with the experiment. The mathematical model captures enough of the physics to approximate the system response and allow generation of an optimally shaped cavity.

\subsection{Optimization of cavity/actuator system}

Multiple optimizations were run with different initial cavity shapes such as straight, tapered, and spear point. For each optimization, the drive voltage remained constant at 90 volts across each layer. The results of the optimization using air as the working fluid are shown in Figure 20 and Figure 21. The cross-section displayed in Figure 20 is distorted, the axes are not plotted equally to allow for modification of the cavity. Each blue control point can be moved by clicking on it with the mouse and dragging to a new location. This lets the user have a qualitative feel for the sensitivity of the solution to small cavity dimensional changes. 
Initial shape black, Mesh red, Control blue

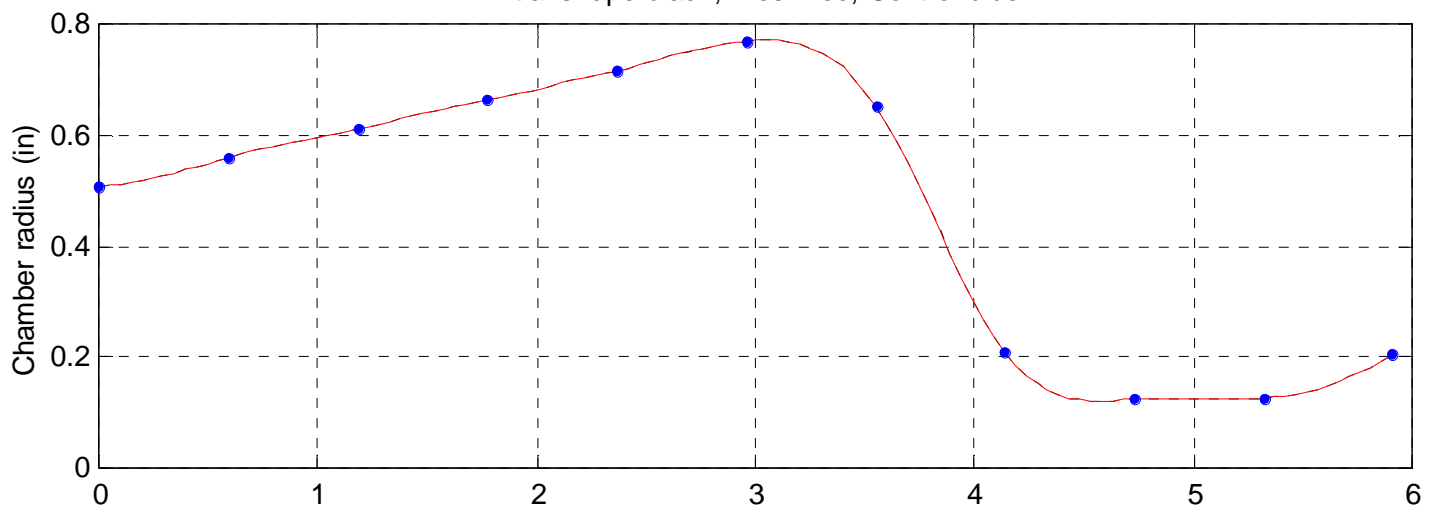

Response at Resonant Modes, 60 piezo, 120 fluid (Air) elements
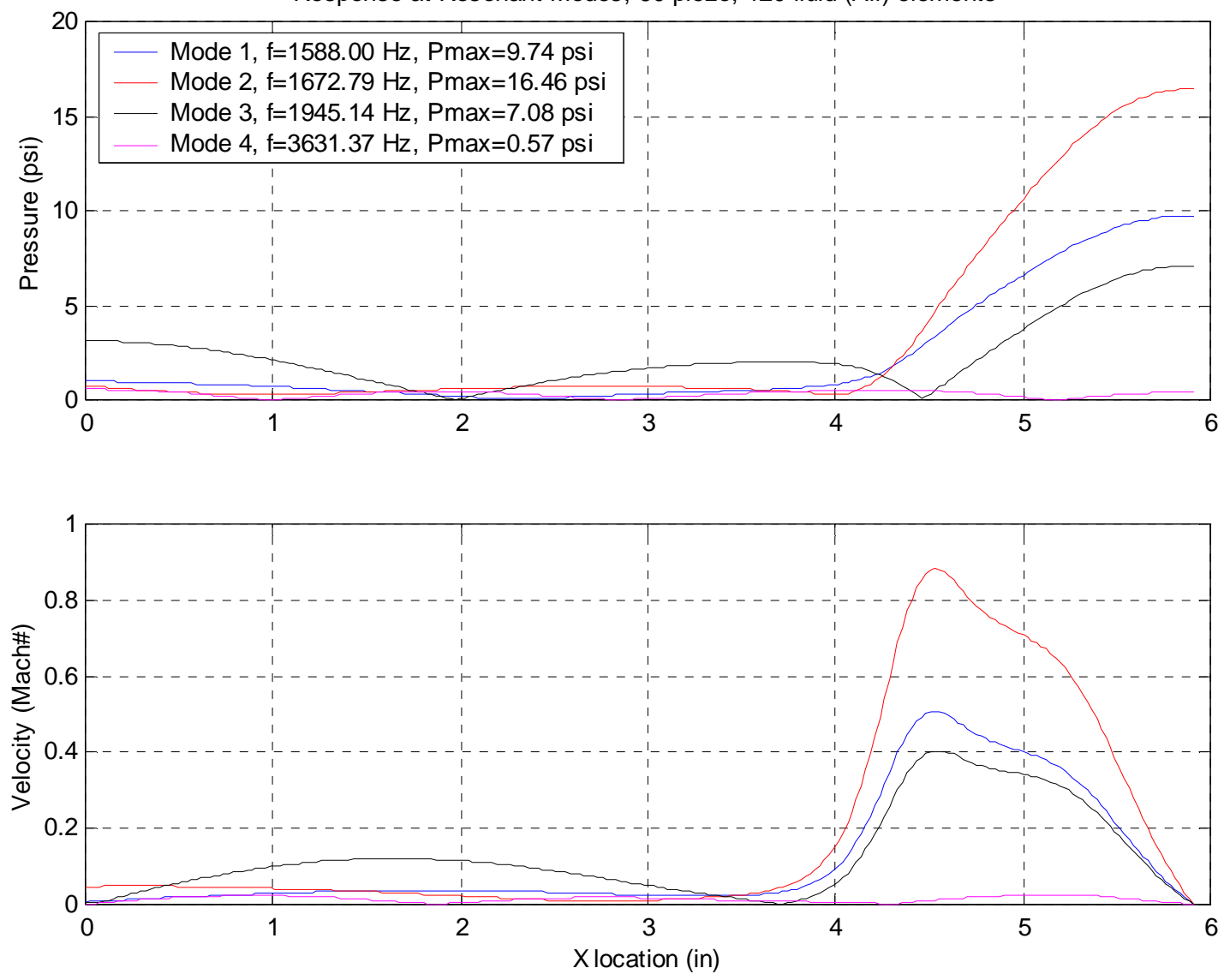

Figure 20 - Matlab output of optimized shape (Air) 

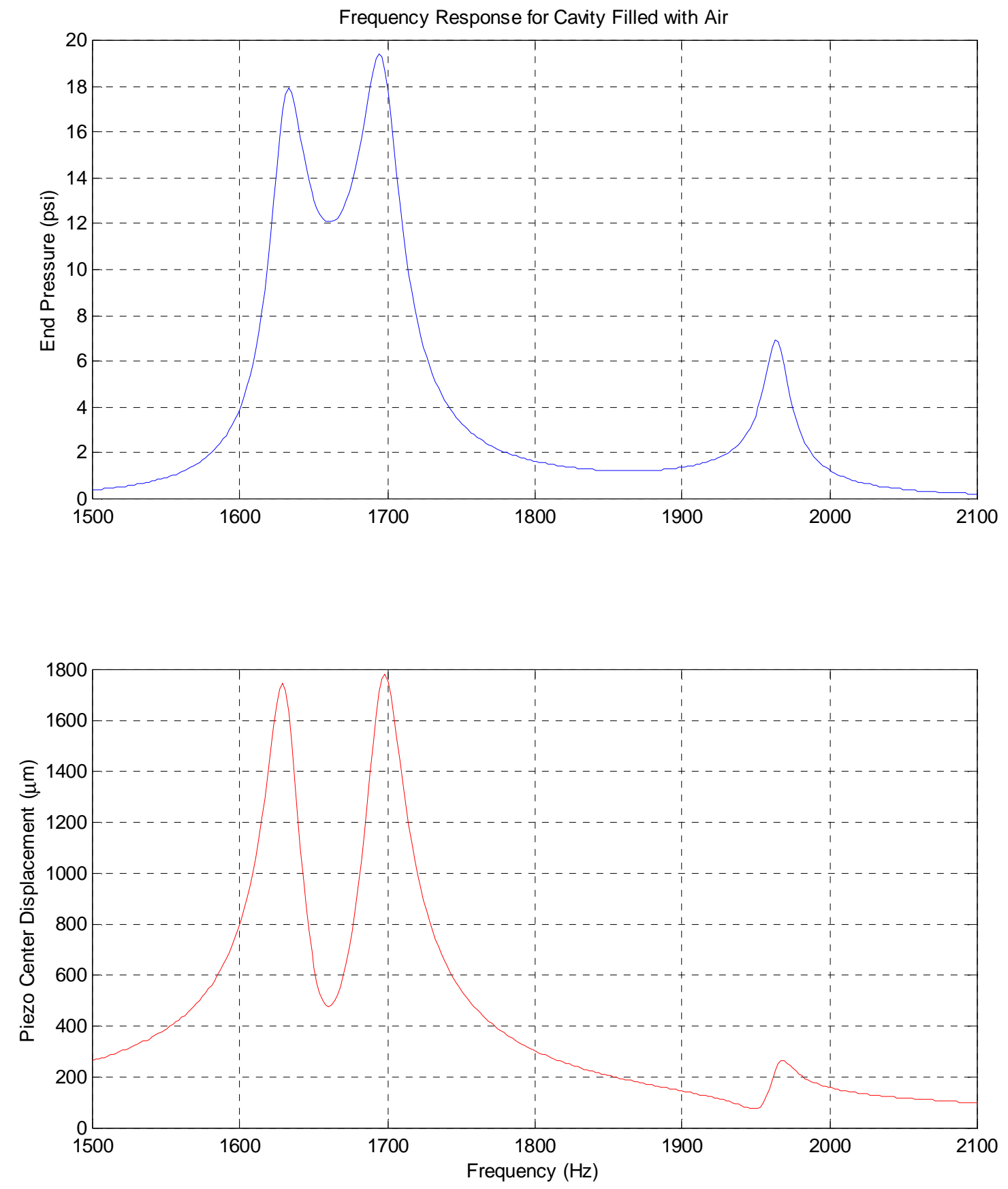

Figure 21 - Matlab frequency response of optimized shape (Air)

Figure 21 shows a spectrum plot of the response. If the application only requires \pm 12 psi, a simple control system could be used as the frequency range is large for this level. 
For the best pressure delivery, a fairly tight controller with feedback is required to maintain operation at the optimum point at the peak of the pressure.

A three-dimensional cross-section of the optimized shape is shown in Figure 22. The shape bears a striking resemblance to a wine bottle.

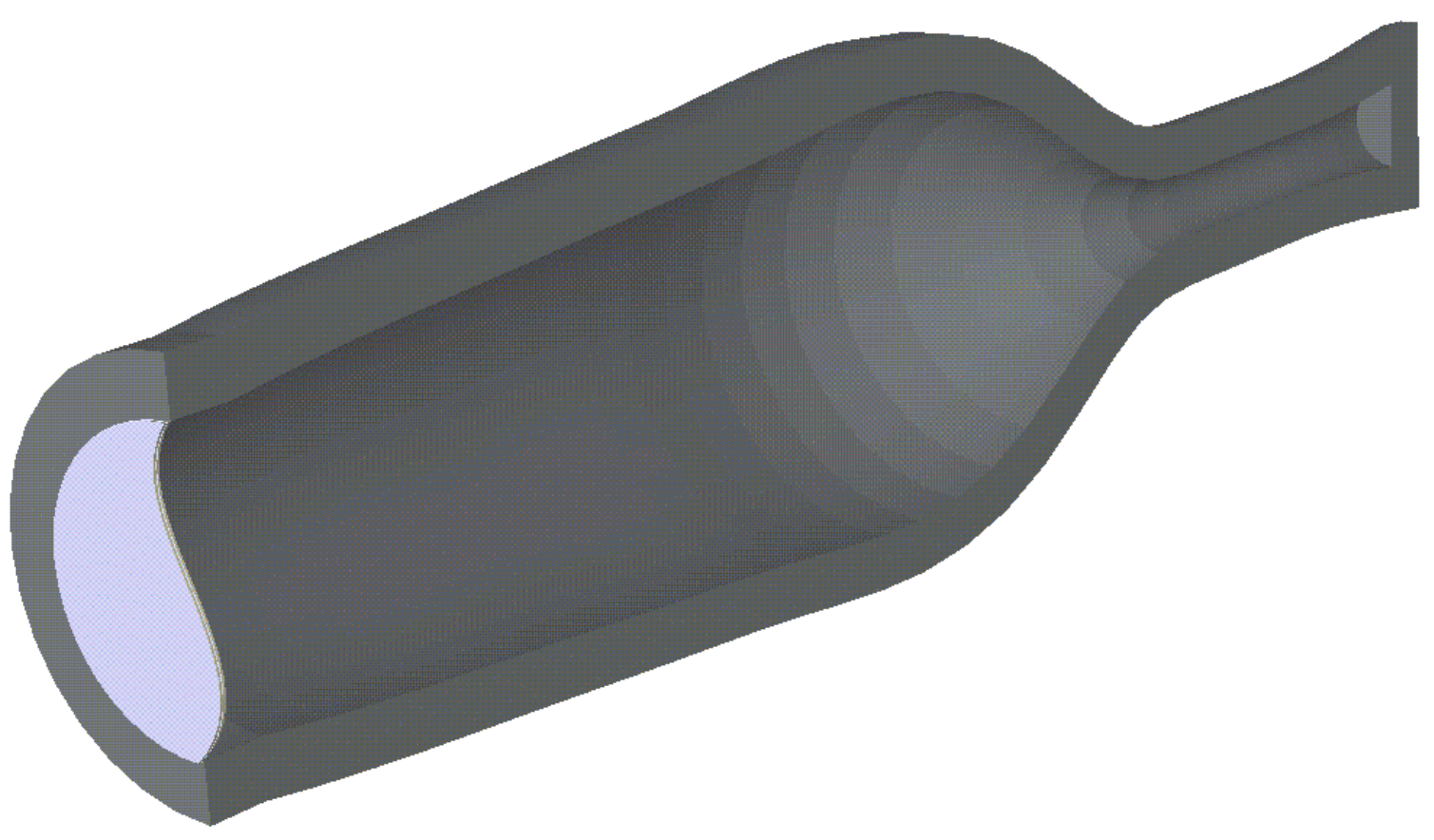

Figure 22 - Cross section of optimized shape (Air)

Using the air solution as a starting point, another optimization was done for R-134A as the working fluid. Mode shapes and frequency response plots follow in Figure 23 and Figure 24. The resulting cavity delivers relatively high pressures, possibly enough to drive a small refrigeration system. 

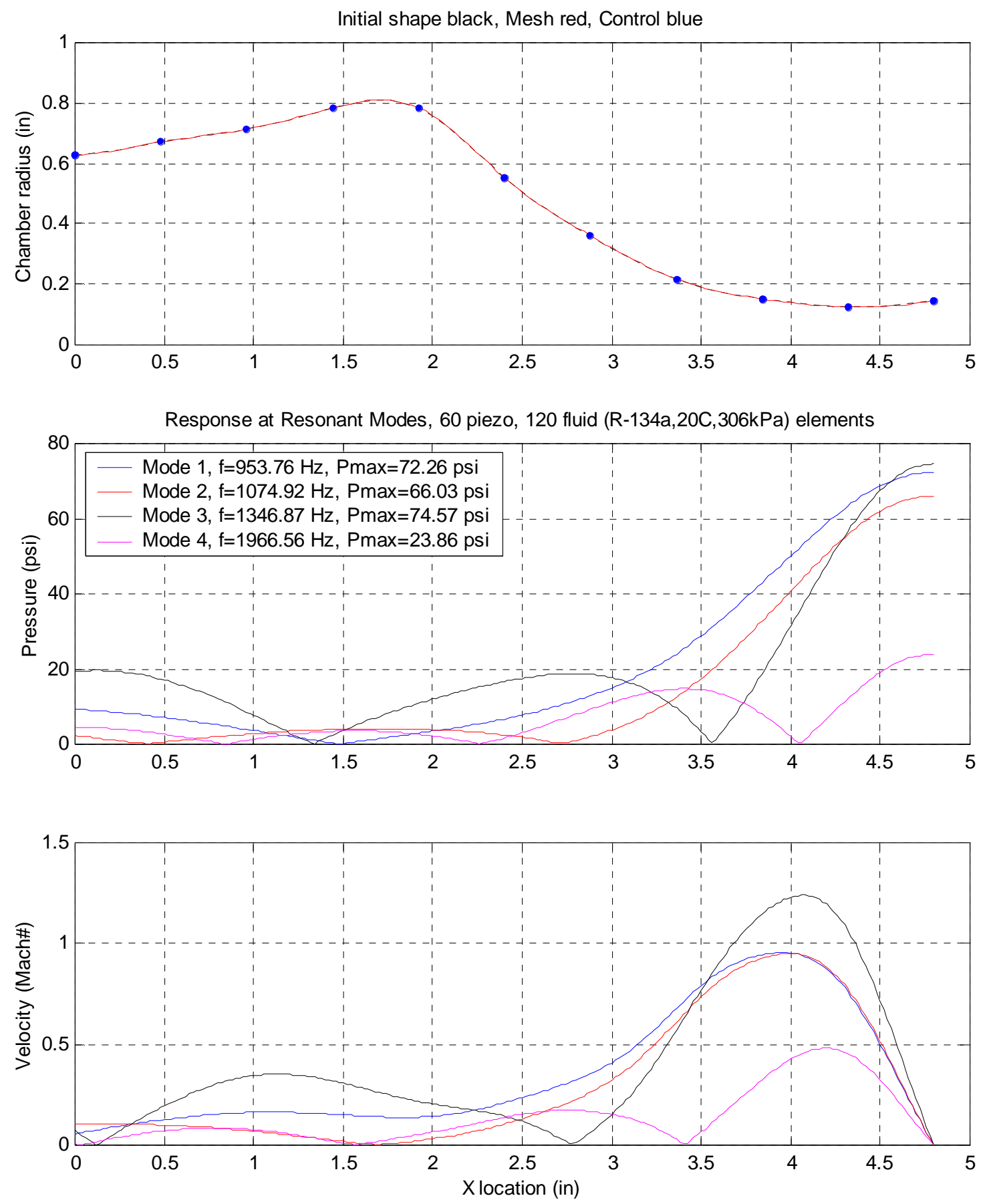

Figure 23 - Matlab output of optimized shape (R-134A) 

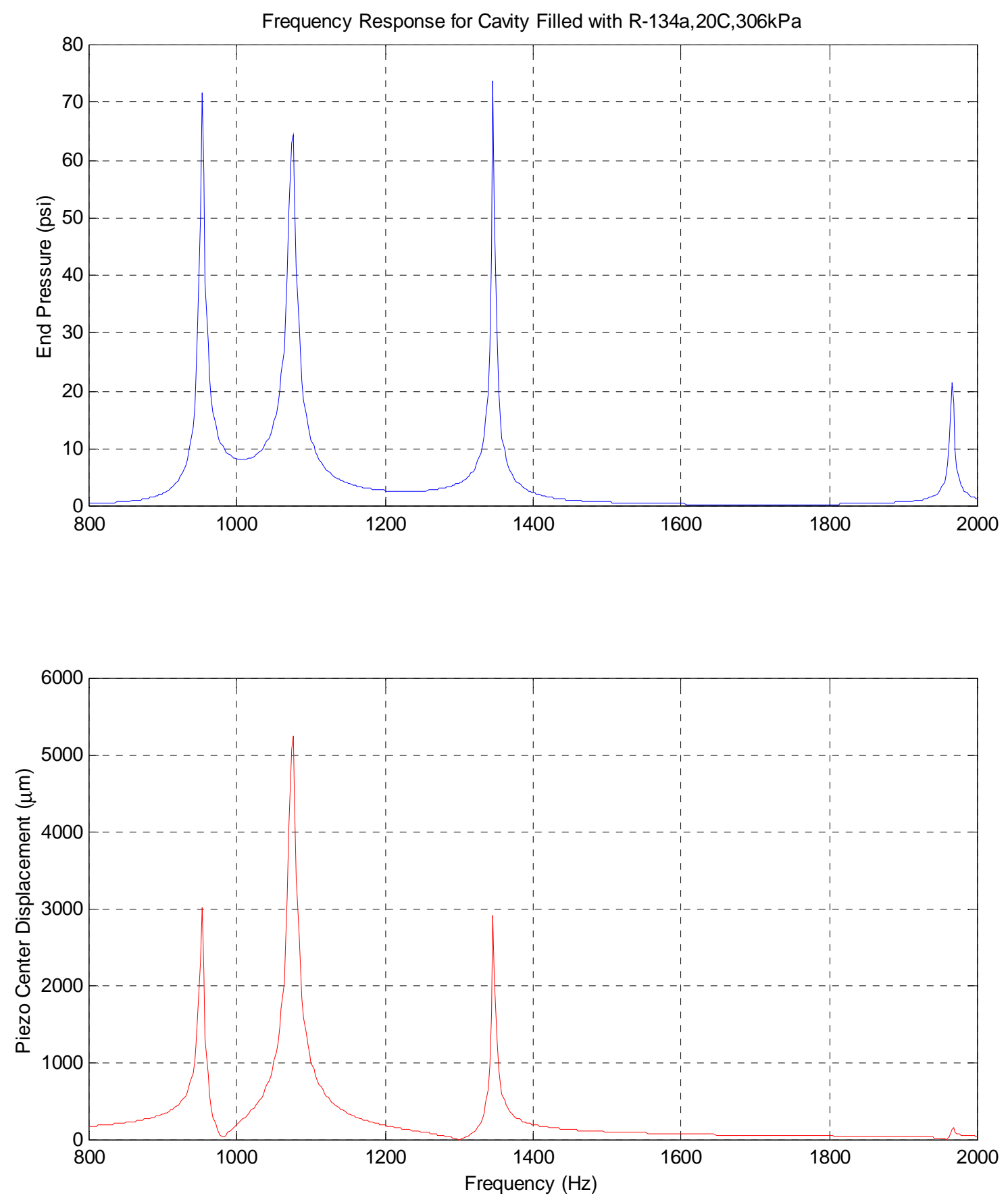

Figure 24 - Matlab frequency response of optimized shape (R-134A) 
The preceding shapes were transferred to ANSYS for verification. The shape was generated in ANSYS using the native cubic spline and the control points as defined by the optimization results.

\subsection{Verification of Optimized Shape}

In the ANSYS model, the walls of the acoustic cavity are assumed rigid and designated as impedance surfaces to include the damping as discussed in section 2.3.1. The piezoelectric bimorph is created and the surface of interaction identified. Plots of the pressure contours are shown below in Figure 25 and Figure 26. Note that the pressure wave is nearly planar at $1719.2 \mathrm{~Hz}$ (Mode 2) but shows considerable two-dimensional effects at $1636.2 \mathrm{~Hz}$ (Mode 1). This likely accounts for some of the differences between the ANSYS and Matlab models. The ANSYS model is a two-dimensional axisymmetric model of the acoustic fluid while the Matlab model is one-dimensional. Table 13 and Table 14 compare and summarize the results of the two solution approaches. 


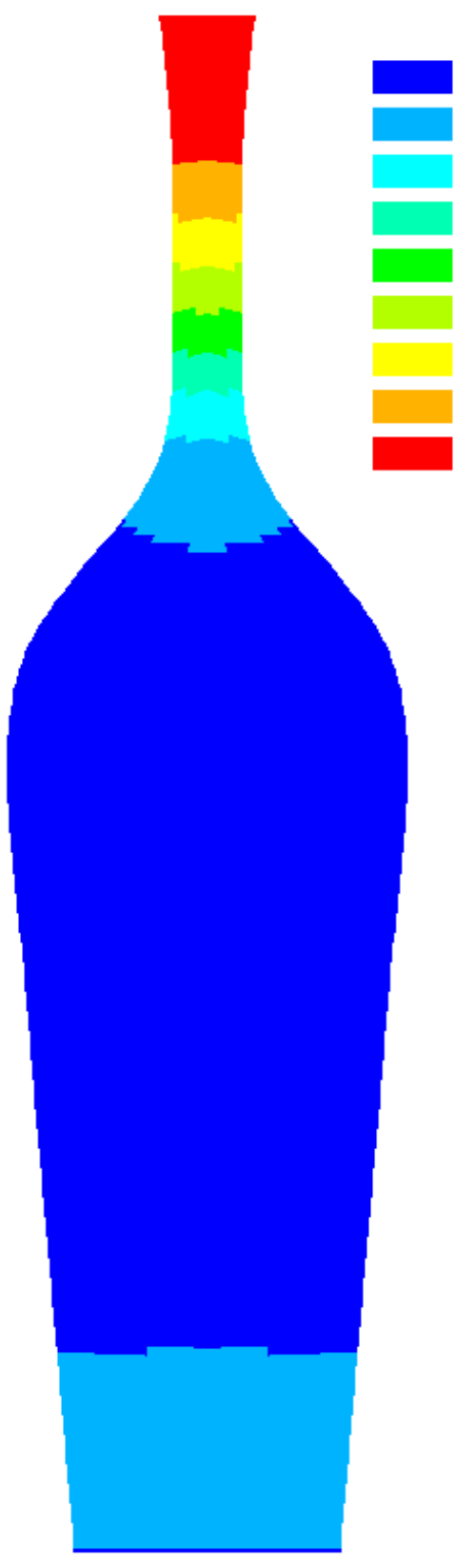

$1616.9 \mathrm{~Hz}$

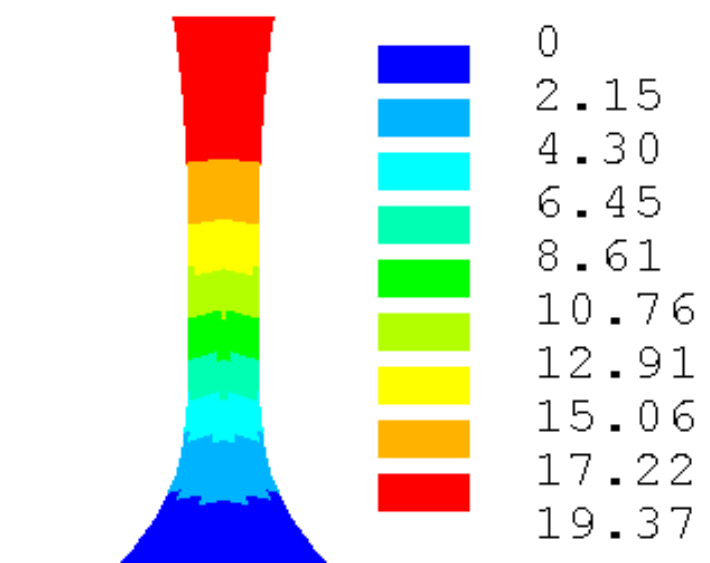

.448

13.22

15.11

17.00

5.669

7.558

9.448

17.00

19.37

4. 30

6.45

8.61

10.76

12.91

15.06
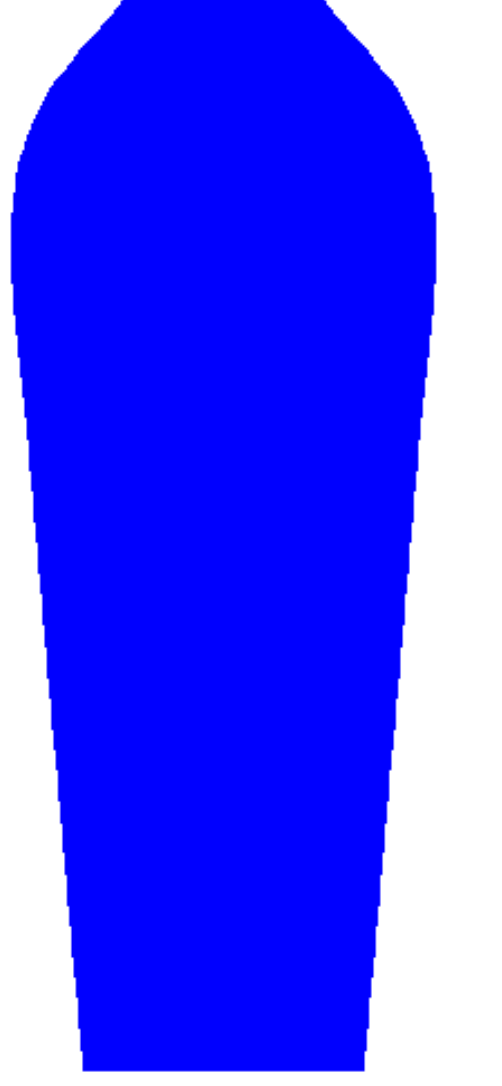

$1678.4 \mathrm{~Hz}$

Figure 25 - Mode 1 and 2 Pressure (psi) in optimized cavity (ANSYS, Air) 


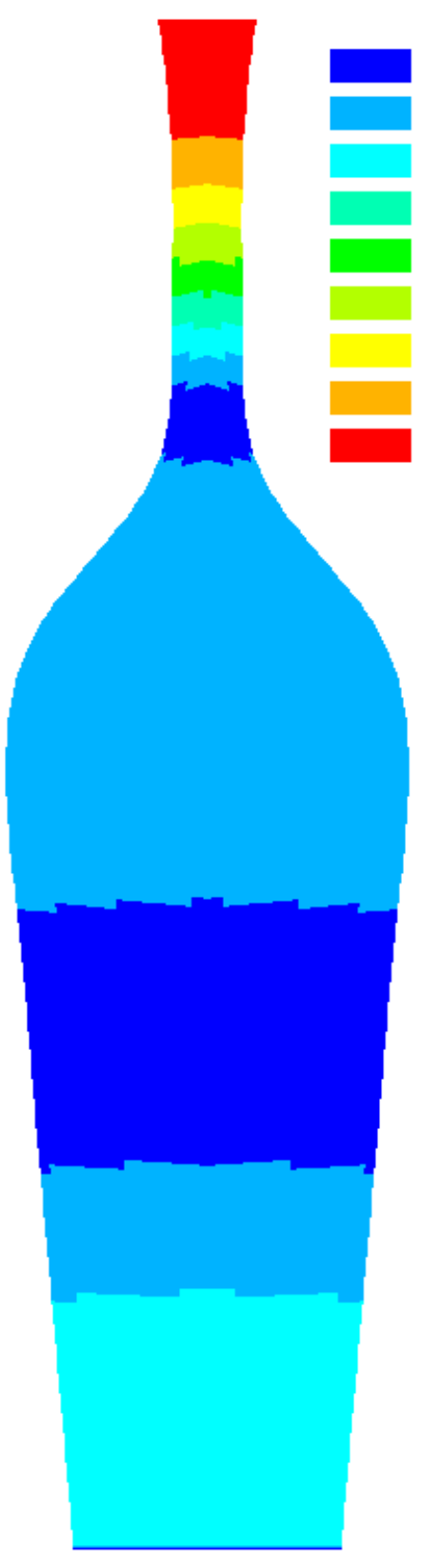

1954.7 Hz

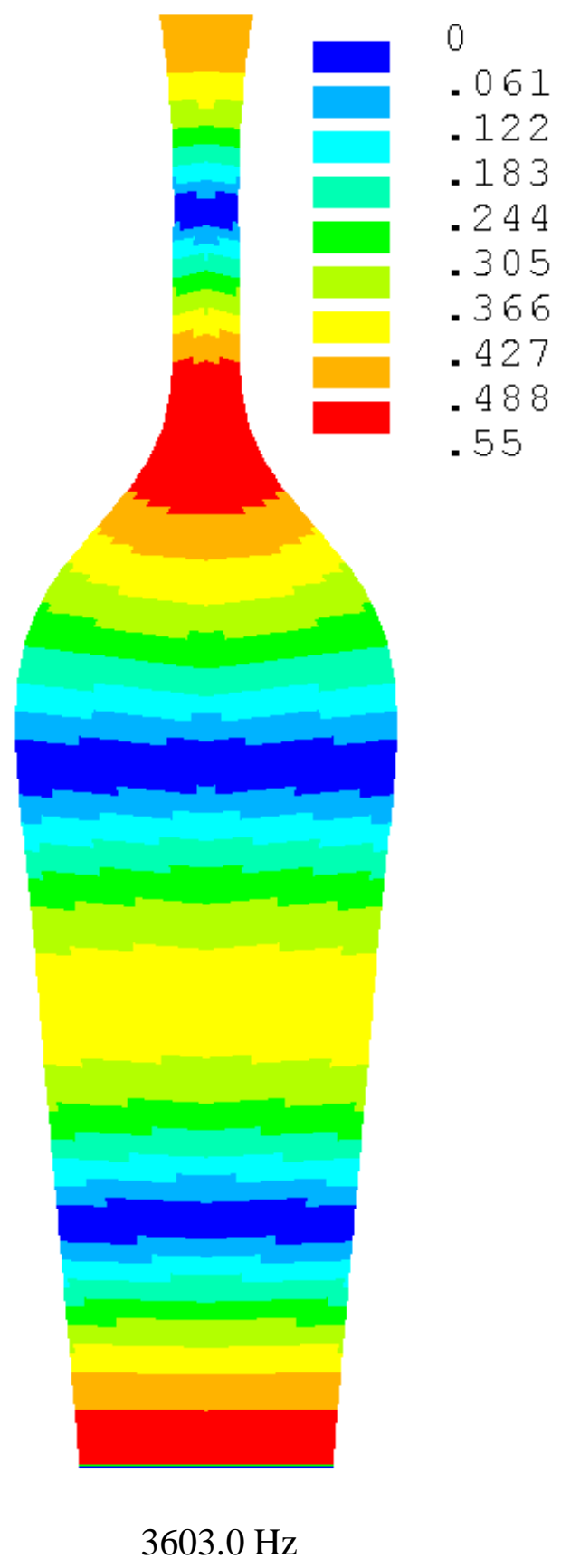

$3603.0 \mathrm{~Hz}$

Figure 26 - Modes 3 and 4 Pressure (psi) in optimized cavity (ANSYS, Air) 
Table 13 - Comparison of Optimized PAC Frequency and Pressure Results (Air)

\begin{tabular}{|c|r|r|r|r|r|r|}
\hline \multirow{2}{*}{$\begin{array}{c}\text { Mode } \\
\text { Number }\end{array}$} & \multicolumn{3}{|c|}{ Frequency $(\mathrm{Hz})$} & \multicolumn{3}{c|}{ End Pressure (psi) } \\
\cline { 2 - 7 } & MATLAB & ANSYS & Difference & MATLAB & ANSYS & Difference \\
\hline 1 & 1632.76 & 1616.90 & $1.0 \%$ & 17.94 & 17.00 & $5.5 \%$ \\
\hline 2 & 1695.23 & 1678.40 & $1.0 \%$ & 19.39 & 19.37 & $0.1 \%$ \\
\hline 3 & 1964.54 & 1954.70 & $0.5 \%$ & 6.963 & 6.774 & $2.8 \%$ \\
\hline 4 & 3634.54 & 3603.00 & $0.9 \%$ & 0.444 & 0.465 & $4.5 \%$ \\
\hline
\end{tabular}

Table 14 - Comparison of Optimized PAC Displacement and Power Results (Air)

\begin{tabular}{|c|r|r|r|r|r|r|}
\hline $\begin{array}{c}\text { Mode } \\
\text { Number }\end{array}$ & \multicolumn{2}{|c|}{ Center point displacement (um) } & \multicolumn{3}{c|}{ Drive Power (watts) } \\
\cline { 2 - 7 } & MATLAB & ANSYS & Difference & MATLAB & ANSYS & Difference \\
\hline 1 & 1654.21 & 1393.49 & $18.7 \%$ & 42.10 & 35.64 & $18.1 \%$ \\
\hline 2 & 1701.46 & 1930.45 & $11.9 \%$ & 44.01 & 49.68 & $11.4 \%$ \\
\hline 3 & 235.01 & 236.28 & $0.5 \%$ & 6.34 & 6.40 & $1.0 \%$ \\
\hline 4 & 19.03 & 19.23 & $1.1 \%$ & 9.96 & 9.88 & $0.8 \%$ \\
\hline
\end{tabular}

Notice the large difference in actuator center displacement in the first two modes. At these large displacements, the assumptions used in the Matlab model become invalid. The ANSYS model does not make the same assumptions, and as such may be considered more correct. Frequency agreement is excellent between the two approaches indicating that the response differences are due to two-dimensional effects and the differences in damping models.

Verification of the optimization using R-134A as the working fluid follows. The shape is very similar, comparisons between ANSYS and the MATLAB solution shown below 

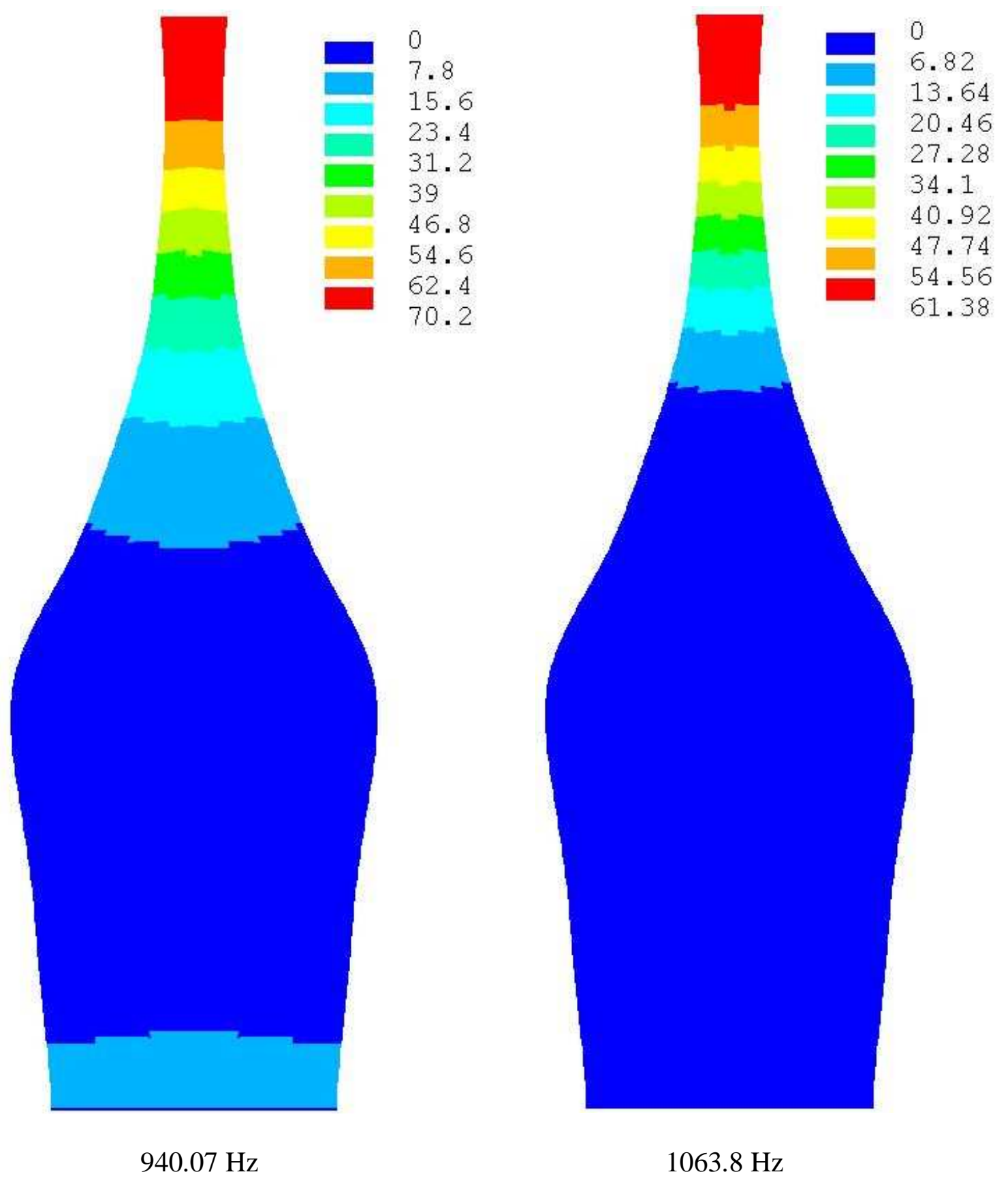

Figure 27 - Mode 1 and 2 Pressure (psi) in optimized cavity (ANSYS, R-134A) 

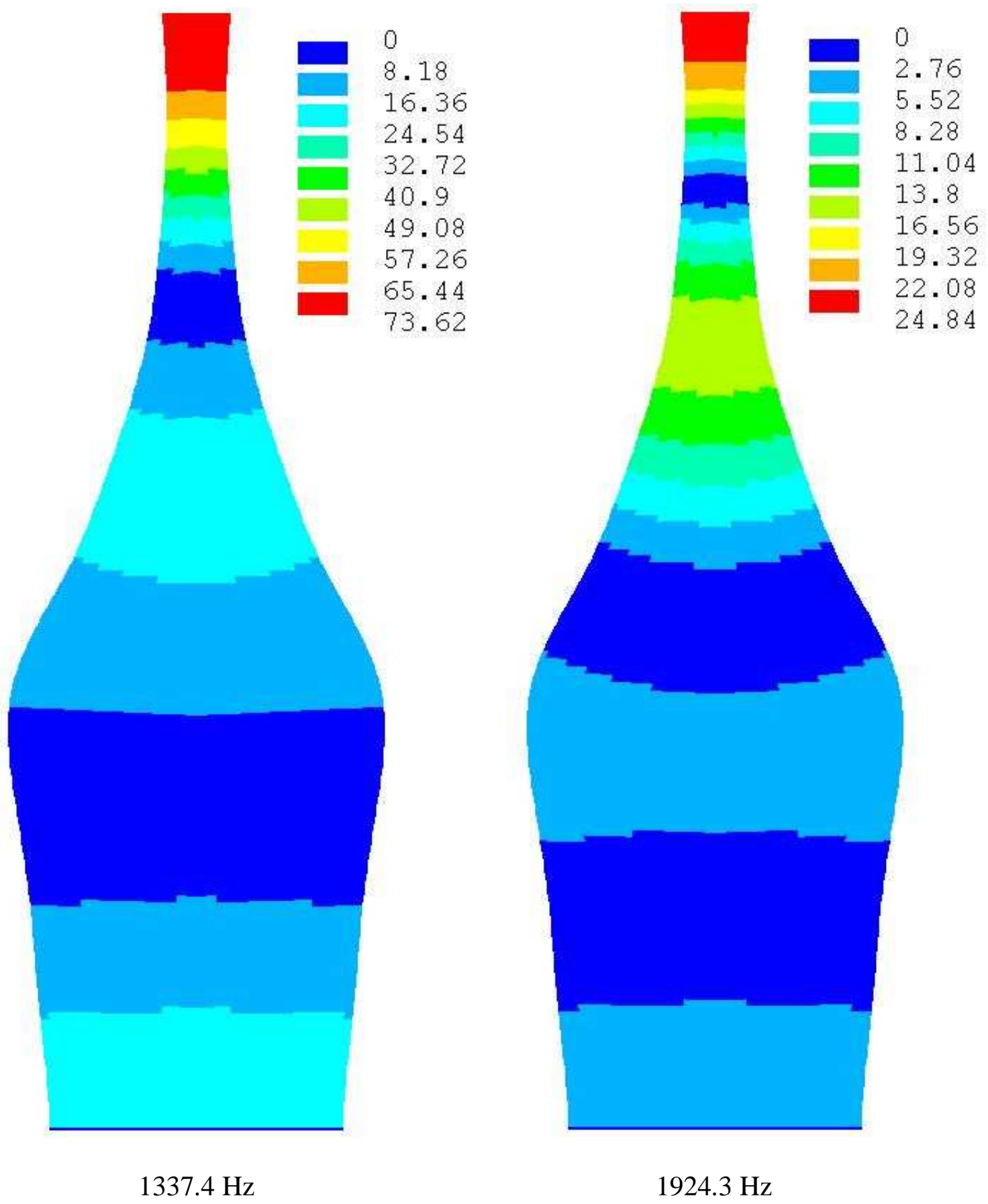

Figure 28 - Mode 3 and 4 Pressure (psi) in optimized cavity (ANSYS, R-134A) 
Table 15 - Comparison of Optimized PAC Frequency and Pressure Results (R-134A)

\begin{tabular}{|c|r|r|r|r|r|r|}
\hline \multirow{2}{*}{$\begin{array}{c}\text { Mode } \\
\text { Number }\end{array}$} & \multicolumn{3}{|c|}{ Frequency (Hz) } & \multicolumn{3}{c|}{ End Pressure (psi) } \\
\cline { 2 - 7 } & MATLAB & ANSYS & Difference & MATLAB & ANSYS & Difference \\
\hline 1 & 953.76 & 940.07 & $1.5 \%$ & 72.26 & 70.09 & $3.1 \%$ \\
\hline 2 & 1074.9 & 1063.8 & $1.0 \%$ & 66.03 & 61.39 & $7.6 \%$ \\
\hline 3 & 1346.9 & 1337.4 & $0.7 \%$ & 74.57 & 73.61 & $1.3 \%$ \\
\hline 4 & 1966.6 & 1924.3 & $2.2 \%$ & 23.86 & 24.80 & $3.8 \%$ \\
\hline
\end{tabular}

Table 16 - Comparison of Optimized PAC Displacement and Power Results (R-134A)

\begin{tabular}{|c|r|r|r|r|r|r|}
\hline \multirow{2}{*}{$\begin{array}{c}\text { Mode } \\
\text { Number }\end{array}$} & \multicolumn{2}{|c|}{ Center point displacement (um) } & \multicolumn{3}{c|}{ Drive Power (watts) } \\
\cline { 2 - 7 } & MATLAB & ANSYS & Difference & MATLAB & ANSYS & Difference \\
\hline 1 & 3002.2 & 2840.6 & $5.7 \%$ & 44.6 & 41.7 & $7.0 \%$ \\
\hline 2 & 5339.0 & 5316.3 & $0.4 \%$ & 88.2 & 87.0 & $1.4 \%$ \\
\hline 3 & 2962.2 & 2809.4 & $5.4 \%$ & 59.7 & 56.2 & $6.3 \%$ \\
\hline 4 & 168.5 & 184.7 & $8.8 \%$ & 9.0 & 9.2 & $1.4 \%$ \\
\hline
\end{tabular}

Figure 27 and Figure 28 show that the R-134A results have more pronounced two-

dimensional effects than the air results. The increased density of R-134A over air allows the chamber to develop higher pressures. Note that the end pressure of 70 psi (mode 1) requires 41.7 watts of electrical power. Referring to Table 10 of the experimental data, 41.7 watts of power delivered to a chamber using ERD produced 90 psi. The paper by Lawrenson, et al. does not specify the electrical power necessary to drive the chamber, only the amount of power delivered to the fluid via the electromechanical actuator. The optimal cavity found here delivers nearly the same amount of pressure for approximately the same power but without the complication of a moving cavity. 


\section{Summary, Conclusions, and Future Work}

The concept of a Piezoelectric Acoustical Compressor was introduced and a finite element model of the system was developed. This model may be used for optimizing the chamber shape and actuator size concurrently. The frequency and pressure predicted by the model is in good agreement with the commercial FEM code ANSYS. The fluid damping model included is in reasonable agreement with one set of experimental measurements found in published literature. An optimal chamber was found that could generate \pm 19 psi at $1700 \mathrm{~Hz}$ for 50 watts of power using air as a working fluid. Another optimal chamber was found using R-134A as working fluid that can generate $\pm 70 \mathrm{psi}$ at $950 \mathrm{~Hz}$ for 42 watts of power.

Future work will be to build and verify the design. As the material properties of piezoelectric materials can vary substantially and fluid properties will change during operation, a control system will be needed to maintain the drive frequency at the optimum point for high-pressure delivery. One-way valves will also need to be added for the compressor to be useful. The results of the experiments can be used to verify the damping model and the piezoelectric drive power model used in this thesis. 


\section{REFERENCES}

Allik, H., and Hughes, T., "Finite Element Method of Piezoelectric Vibration", International Journal for Numerical Methods in Engineering, Vol. 2, pp. 151-157, 1970

American National Standard, IEEE Standard on Piezoelectricity, ANSI/IEEE STD $176,1987$.

ANSYS Theory Reference, Release 5.3, SAS IP, Inc., 1996

Bathe, K., Finite Element Procedures, Prentice Hall, Englewood Cliffs, NJ, 1996

Baz, A., "Active Noise Control of Piston-Cavity Systems", Adaptive Structures and Material Systems, pp. 121-131, 1997

Baz, A., "Proposal for a Piezoelectric Acoustic Compressor", Unpublished work, 2000

Baz, A., Vibration Damping, Class Notes, Spring 2001.

Berlincourt, D., Krueger, H., Near, C., "Technical Publication TP-226, Properties of Piezoelectricity Ceramics", Morgan Electro Ceramics, Published on company website, www.morgan-electroceramics.com.

Bishop, R., "Standing Wave Pump”, U.S. Patent Number 6,079,214, June 25, 2000.

Dobrucki, A., and Pruchnicki, P., "Theory of Piezoelectric Axisymmetric Bimorph", Sensors and Actuators A, Vol. 58, pp. 203-212, 1997.

EES, Engineering Equation Solver, v4.994, F-Chart Software, 1999

Everstine, G., “A Symmetric Potential Formulation for Fluid-Structure Interaction”, $J$. of Sound and Vibration, Vol. 79, No.1, pp. 157-160, 1981.

Hoffelner, J., Landes, H., Kaltenbacher, M., and Lerch, R., "Finite Element Simulation of Nonlinear Wave Propagation in Thermoviscous Fluids Including Dissipation", IEEE Transactions on Ultrasonics, Ferroelectrics, and Frequency Control, Vol. 48, No.3, pp. 779-786, 2001.

Ilinskii, Y., Lipkens, B., Lucas, T. S., Van Doren, T. W., and Zbolotskaya, E., "NonLinear Standing Waves in an Acoustical Resonator", Journal of the Acoustical Society of America, Vol. 104, No. 5, pp 2664-2674, 1998. 
Ilinskii, Y., Lipkens, B., and Zbolotskaya, E., "Energy Losses in an Acoustical Resonator", Journal of the Acoustical Society of America, Vol. 109, No. 5, pp 18591870, 2001.

Kays, W., and Crawford, M., Convective Heat and Mass Transfer, McGraw-Hill, Inc., 1993.

Kinsler, L., Frey, A., Coppens, A., and Sanders, J., Fundamentals of Acoustics, John Wiley \& Sons, Inc., 1982.

Lawrenson, C., Lipkens, B., Lucas, T. S., Perkins, D., and Van Doren, T. W., "Measurements of Macrosonic Standing Waves in Oscillating Closed Cavities", Journal of the Acoustical Society of America, Vol. 104, No. 2, pp 623-636, 1998.

Ling-Hui, He, "Axisymmetric Response of Circular Plates with Piezoelectric Layers: An Exact Solution”, International Journal of Mechanical Sciences, Vol. 40, No. 12, pp. 1265-1279, 1998.

Lucas, T. S., "Compression-Evaporation Cooling System Having Standing Wave Compressor", U.S. Patent Number 5,357,757, October 25, 1994.

Lucas, T. S., Van Doren, T. W., "Resonant Macrosonic Synthesis", U. S. Patent Number 5,515,684, 1996.

Mehta, R., and Bradshaw, P., "Technical Notes: Design Rules for Small Low Speed Wind Tunnels", The Aeronautical Journal of the Royal Aeronautical Society, November, 1979.

Meirovitch, L., Fundamentals of Vibrations, McGraw-Hill, New York, NY, 2001

Morse, P., and Ingard, K., Theoretical Acoustics, McGraw-Hill, New York, NY, 1968

Nashif, A., Jones, D., and Henderson, J., Vibration Damping, John Wiley \& Sons, Inc., 1985.

Near, C., "Piezoelectric Actuator Technology”, SPIE Vol. 2717, 1996.

Olson, L., Bathe, K., "Analysis of Fluid-Structure Interactions. A Direct Symmetric Coupled Formulation Based on Fluid Velocity Potential", Computers and Structures, Vol. 21, No. 1/2, pp. 21-32, 1985.

Page, N., Mee, D., "Wall Effects on Sound Propagation in Tubes", Journal of Sound and Vibration, Vol. 93, No. 4, pp. 473-380, 1984 
Rodarte, E., Singh, G., Miller, N., and Hrnjak, P., "Sound Attenuation in Tubes Due to Visco-Thermal Effects", Journal of Sound and Vibration, Vol 231, No. 5, pp. 1221$1242,2000$.

Tijdeman, H., "On the Propagation of Sound Waves in Cylindrical Tubes", Journal of Sound and Vibration, Vol. 39, No. 1, pp. 1-33, 1975

Zuckerwar, A., Meredith, R., "Low Frequency Absorption of Sound in Air", Journal of the Acoustical Society of America, Vol. 78, No. 3, pp 946-955, 1985. 